\title{
THE IMPACT OF MOTION CUES ON MEMORY FOR OBJECT LOCATION AND APPEARANCE
}

\author{
by \\ Chris M. Nicholson \\ A thesis submitted to \\ the Faculty of Graduate and Postdoctoral Affairs \\ in partial fulfillment of the requirements for the degree of: \\ Master of Arts \\ in \\ Psychology \\ Carleton University \\ Ottawa, Canada \\ (C)2014 \\ Chris M. Nicholson
}




\begin{abstract}
Two experiments were conducted to investigate the notion that working memory is fractionated into visual and spatial components by demonstrating that a motion discrimination task selectively interferes with the spatial component. Also, because the motion cues used in the motion discrimination task were similar to those used in flight simulators, an attempt was made to further understand which aspects of working memory are required to monitor those motion cues. These experiments examined participants' ability to remember either the location or the appearance of visual stimuli while concurrently discriminating between left/right motion cues produced by a motion seat. Motion cues occurred either during stimulus encoding (E1) or retention (E2) of the visual stimuli. The ability to remember the location of visual stimuli was significantly impaired by motion cues presented during either encoding or retention. In contrast, the motion cues did not interfere with memory for the stimulus' appearance. This study supports the multi-component model of working memory and the notion that encoding/retention of location and appearance information is served by separable mechanisms in working memory. The finding that there is a cognitive cost of processing of visual-spatial information while interpreting motion cues, highlights the importance of including some form of motion cueing in flight simulators to more accurately represent the true mental demands of dynamic flight.
\end{abstract}




\section{Acknowledgments}

I would like to acknowledge the following people for their invaluable assistance:

My supervisor Dr. (Sr.) Chris Herdman for his truly expert guidance and knowledge and for building an amazing lab, filled with topnotch people.

Dr. Matt Brown for his precision editing, helpful feedback and guidance (academically as well as personally), and for just being there.

Andy, Jon, and Travis for their invaluable technical ability with computer stuff and things - without you no science would happen.

My committee for their participation and constructive feedback. Specifically, Dr. Jo-Anne LaFevre for her initial guidance with my prospectus and through to the final product.

My family and friends for their unending support and confidence. In particular, my selfless editor, you will be kept on - Allons-y! 


\section{Table of Contents}

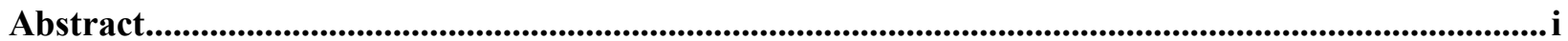

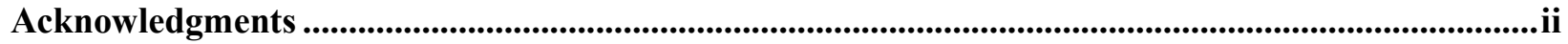

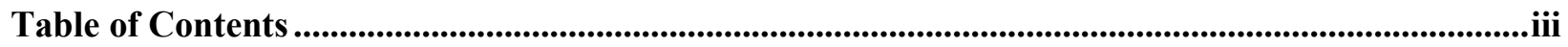

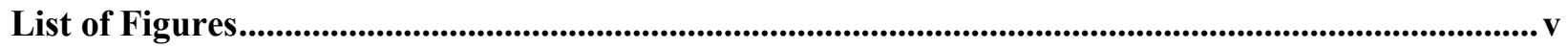

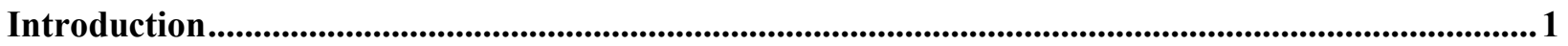

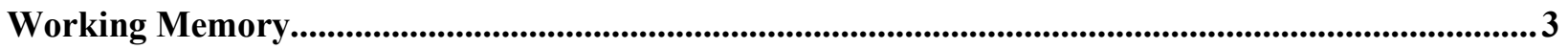

Motion Perception and Visual-Spatial WM ..............................................................................................9

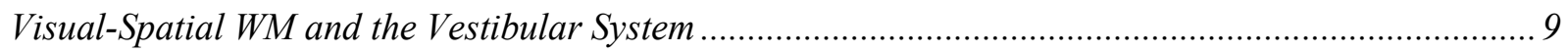

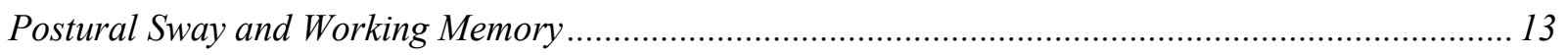

Effects of Eye and Limb Movements on Spatial Working Memory ............................................. 17

Simulated Motion and Spatial Working Memory ..................................................................... 20

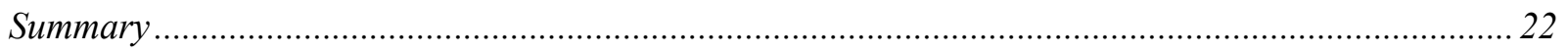

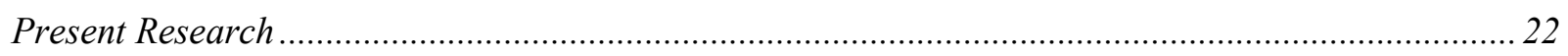

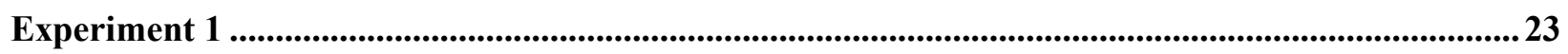

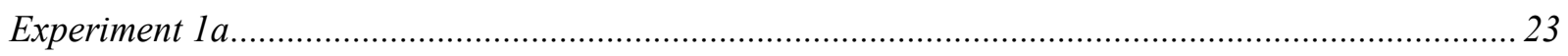

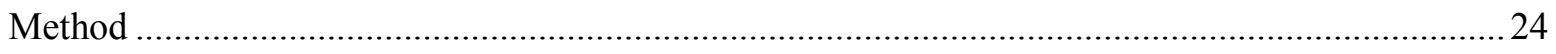

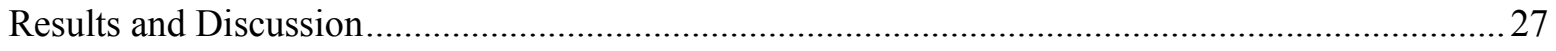

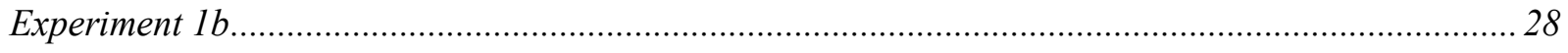

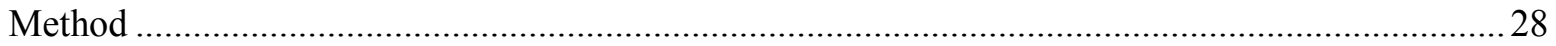

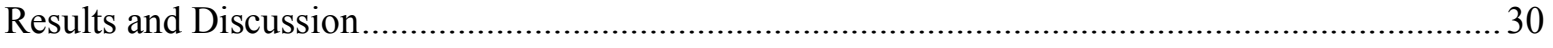

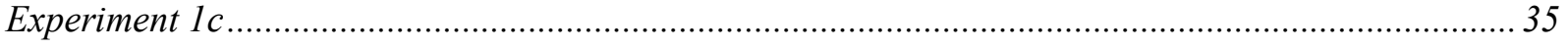

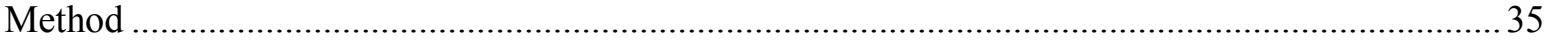




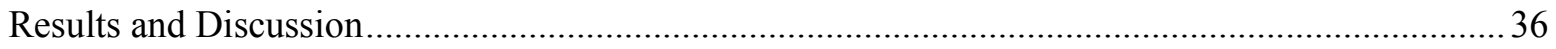

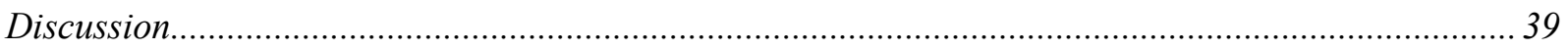

Experiment 2 ..................................................................................................................................................................... 40

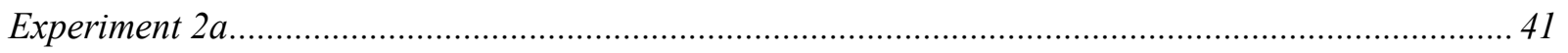

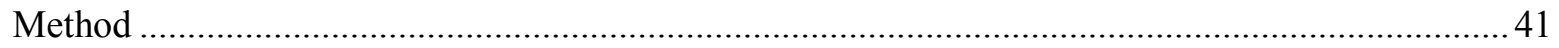

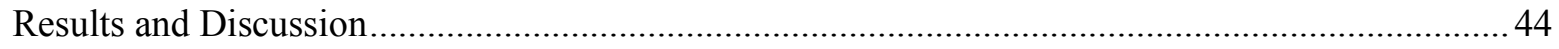

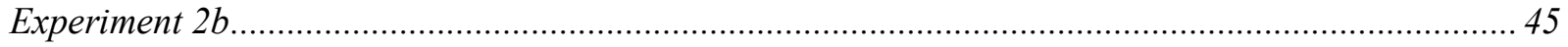

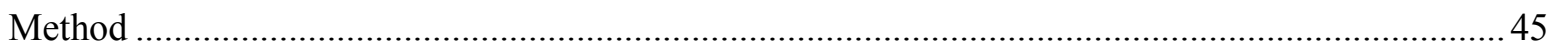

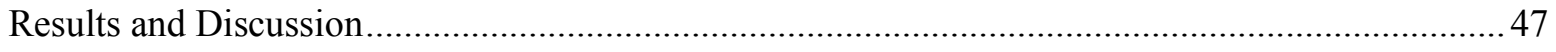

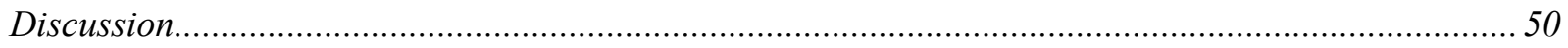

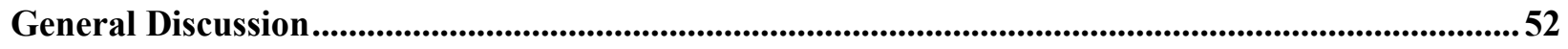

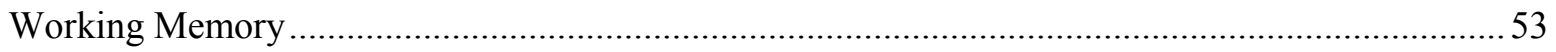

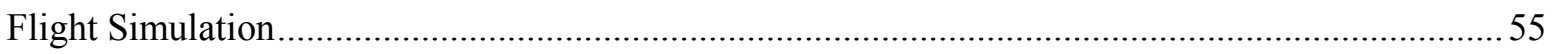

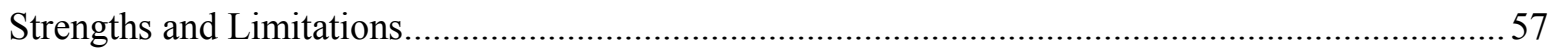

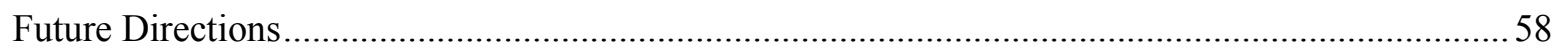

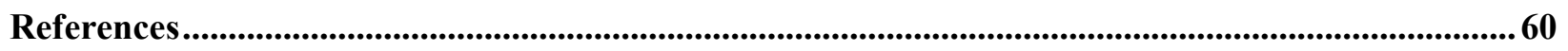

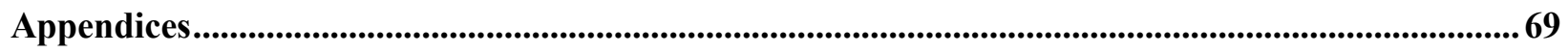

Appendix A: Participant Informed Consent ........................................................................................................70

Appendix B: Participant Demographic Questionnaire ................................................................................72

Appendix C: Participant Debrief......................................................................................................................... 73 


\section{List of Figures}

Figure 1. Diagram of Baddeley's (1984) multi-component model of WM. .............................. 4

Figure 2. An updated version of the multi-component model of WM..................................... 5

Figure 3. Examples of a "P" from each of the four category dimensions used as stimuli in

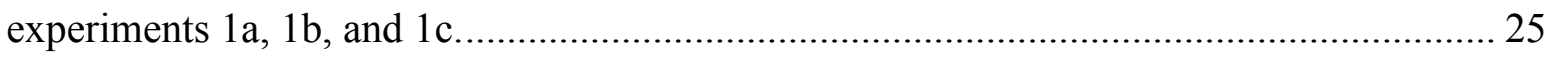

Figure 4. Example of a "same" spatial or visual trial sequence from Experiments 1a, 1b, and 1c.

Figure 5. Mean accuracy on the motion cue task as a function of Memory Task in Experiment

1b. Error bars are $95 \%$ confidence intervals.

Figure 6. Mean response time (in ms) on the memory task as a function of Motion Cueing and

Memory Task in Experiment 1b. Error bars are 95\% confidence intervals. 33

Figure 7. Mean accuracy on the memory task as a function of Motion Cueing and Memory in

Experiment 1b. Error bars are 95\% confidence intervals.

Figure 8. Mean response time (in ms) on the memory task as a function of Motion Cueing and

Memory Task in Experiment 1c. Error bars are 95\% confidence intervals.

Figure 9. Mean accuracy (\% correct) on the memory task as a function of Motion Cueing and

Memory Task in Experiment 1c. Error bars are 95\% confidence intervals. 38

Figure 10. Example of a "same" spatial or visual trial sequence from Experiments 2a and 2b... 44

Figure 11. Mean response accuracy (\% Correct) on the memory tasks as a function of Memory

Task in Experiment 2a. 45

Figure 12. Mean response time (in $\mathrm{ms}$ ) on the memory task as a function of Motion Cueing and

Memory Task in Experiment 2b. Error bars are 95\% confidence intervals. 48 
Figure 13. Mean accuracy ( $\%$ Correct) on the memory task as a function of Motion Cueing and Memory Task in Experiment 2b. Error bars are 95\% confidence intervals.......................... 50 


\section{Introduction}

Flight simulators are used extensively in the aviation industry to train pilots. Simulator technologies have evolved to include a range of training systems from part-task trainers to full flight, Level D simulators. Part-task trainers are reproductions of specific panels and functional components of the flight deck. Part-task trainers are used for procedural training and familiarization with flight instruments. In contrast, Level D full flight simulators are the highestrated class of simulators and they must include an exact reproduction of the simulated aircraft's flight deck, advanced visuals, realistic sound, and a six-degree-of-freedom (6-DOF) motion system (Federal Aviation Administration, 2013). Used in the context of a full training program, flight time accumulated in a Level D simulator can be logged as flight hours equivalent to those gained in the actual aircraft. One key requirement of a Level D simulator is that it must simulate an aircraft's motion. The objective of the present research was to examine the role of spatial working memory in perceiving and responding motion cues.

The requirement to include motion in Level $\mathrm{D}$ simulators is based on the assumption that to maximize training, motion information that is perceived by pilots when flying a real aircraft must also be presented during simulated flight (Burki-Cohen, Soja, \& Longridge, 1998). Including motion in a flight simulator is costly and complex. Simulating motion involves the use

of 6-DOF systems that use pitch, roll, yaw, and vertical and longitudinal acceleration to represent the complex motions experienced during flight. If not implemented correctly, simulated motion can negatively impact performance. For example, poorly implemented motion can induce simulator sickness (Been-Lirn Duh, Parker, Philips, \& Furness, 2004; Stein \& Robinski, 2012; Wertheim, 1998), which can interfere with training objectives. Poorly implemented motion may also train pilots to detect and utilize motion information that is not informative, or worse, are 
incorrect. For example, if an aircraft experiences a lack of airflow over the wings (stall), the loss of lift can cause control input responses to be delayed. These slow control responses may not be noticed in a simulator if the appropriate motion information is not presented. More importantly, pilots may not recognize slow controls in a real aircraft stall if they have not felt the stall previously in a flight simulator.

There is little scientific evidence to support the claim that including motion in flight simulators facilitates transfer of training (Bürki-Cohen et al., 2000; de Winter, Dodou, \& Mulder, 2012; McCauley, 2006). In research where reasonably valid measurement has been accomplished, the bulk of the evidence suggests that the inclusion of motion may not provide a significant benefit to simulator training (de Winter et al., 2012; McCauley, 2006). However, these studies have typically focused on examining how motion affects performance on tasks that directly relate to motion, or "first-order" task. First-order tasks are often complex. For example, measurement of flight control is a first-order task that includes airspeed, altitude, heading, and in some cases recovery from an external event (e.g., responding to changes in environmental conditions). These components of flight control are inter-dependent and cannot be considered alone: an increase in airspeed may be caused by a decrease in altitude that itself was precipitated by a change in heading or flight into turbulence (Burki-Cohen et al., 1998; Burki-Cohen et al., 2000; de Winter et al., 2012). Given the complexity and interdependence of tasks, it is challenging to isolate and measure aspects of performance that may be directly affected by motion.

One perspective is that motion may affect the ability of pilots to perform tasks that are associated with aircraft operation but which are secondary to flight control (e.g., remembering and tracking elements on a visual display). The influence of motion would be indirect insofar as 
motion information is not required to perform second-order tasks. On this view, the cognitive demands associated with monitoring and responding to motion may deplete the availability of cognitive resources that are required to perform second-order tasks. One cognitive mechanism that might be involved in the perception of motion is working memory (WM). Research has shown a link between WM and various forms of motion information, including postural sway (Lajoie, Teasdale, Brad, \& Fleury, 1993; Maylor \& Wing, 1996; Ramenzoni, Riley, Shockley, \& Chiu, 2007), angular rotation (Yardley, Gardner, Lavine, \& Gresty, 1999; Yardley et al., 2002), maintenance or regaining of orientation (Gresty \& Golding, 2009; Gresty et al., 2003; Webb et al., 2011), and unattended motion (Yardley et al., 2002). These studies suggest that WM capacity is required to perceive and process many forms of self-motion and that there is likely a meaningful connection between WM and motion.

The objective of the present research was to examine the connection between the perception of motion and WM. A better understanding of how motion cues are perceived and processed in WM will lead to more informed decisions about the implications of including motion in simulation-based training.

\section{Working Memory}

Baddeley and colleagues' multi-component model is the most pervasive framework used to study WM (Baddeley, 1984; 1986; 1988; 1992; 2012; Baddeley \& Hitch, 1974). Contrasted by models of WM that propose a single general resource (Engle, Kane, \& Tuholski, 1999; Vergauwe, Barrouillet, \& Camos, 2009), Baddeley and colleagues' model consists of multiple components that handle a range of specific tasks (Baddeley, 1992; 1996). The most basic form of the multi-component model of WM is shown in Figure 1. The model contains three components: the Central Executive, Phonological Loop, and Visual-Spatial Sketchpad (VSSP). 


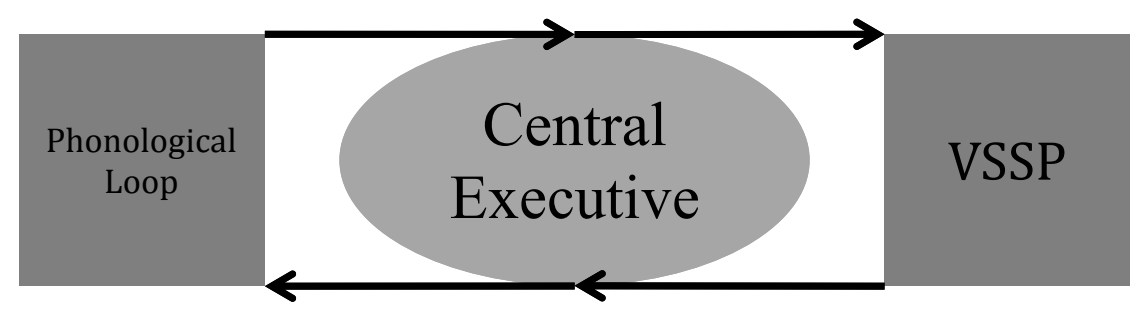

Figure 1. Diagram of Baddeley's (1984) multi-component model of WM.

The central executive is assumed to control the assignment and maintenance of attention, task switching, inhibition, and the encoding and retrieval of information into/from long-term memory (Baddeley, 1996; Logie, 2011). The central executive is the link between WM's two subsystems - the phonological loop and the VSSP.

The phonological loop has been the most extensively studied of the two subsystems (Baddeley, 1992; Vicari, Bellucci, \& Carlesimo, 2003), undoubtedly because it has been easier to assess due to its strong involvement in language. Using dual-task paradigms, researchers have measured the impairment of verbal memory caused by concurrent performance of articulatory suppression tasks, which require the continual repetition of a word (e.g., "the, the, the"). The articulatory suppression effect shows that sub-vocal rehearsal is required to refresh and hold verbally encoded material in WM (Cocchini et al., 2002; Logie \& Baddeley, 1987; Rudkin, Pearson, \& Logie, 2007). Research suggests that the phonological loop is comprised of two mechanisms, one for articulatory storage and one for articulatory rehearsal (Logie, 2011).

The VSSP subsystem has been acknowledged as a potential link between WM and motion perception/processing (Smith, Darlington, \& Zheng, 2010; Thomas \& Seiffert, 2010). The VSSP is thought to be involved in both the storage and processing of an object's colour, shape, spatial location, orientation, and movement (Baddeley, 1996; Vicari, Bellucci, \& Carlesimo, 2003). Like the multi-component model's other components, the VSSP has finite 
resources and has been shown to be limited to processing about four units of information at a time (Woodman, Vecera, \& Luck, 2003; Zimmer, Munzer, \& Umla-Runge, 2010). The VSSP was initially viewed as a unitary subsystem for the storage of visual and spatial information (Baddeley, 1996). However, it has been suggested that the VSSP may be fractionated into two domain-specific subcomponents - a visual cache and an inner scribe (Logie, 1995; 2011). The visual cache is assumed to store visual information, whereas the scribe processes spatial information. An updated schematic of the multi-component model of WM can be seen in Figure 2.

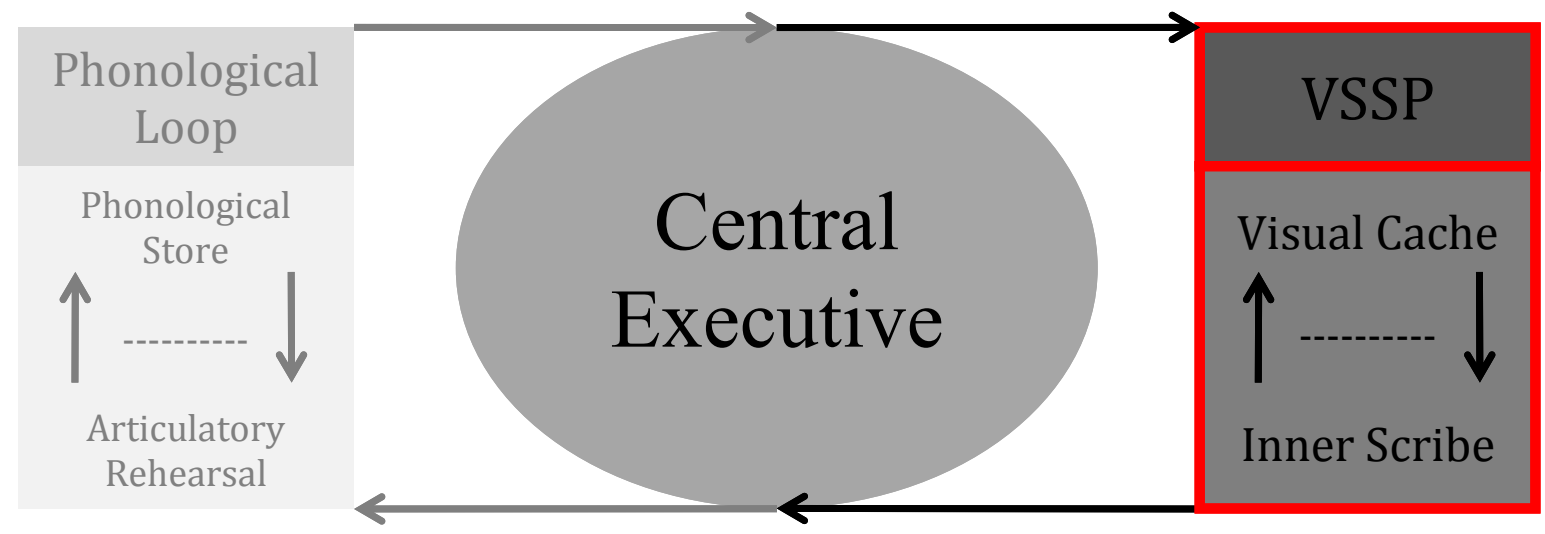

Figure 2. An updated version of the multi-component model of WM.

When people attend to visual stimuli there are two main features they must consider: the stimulus' appearance/form and its location in space. Remembering the appearance of an object is fundamentally different than remembering its location (Sala, Gray, Baddeley, Allamano, \& Wilson, 1999; Farmer, Berman, \& Fletcher, 1986; Hale et al., 1996; Postle, D’Esposito, \& Corkin, 2005; Tresch, Sinnamon, \& Seamon, 1993). This appearance/location distinction has been tested in behavioural studies through the use of dual-task experiments. Darling, Sala, and Logie (2009) developed a memory paradigm to test memory for the spatial location or the appearance of a visual stimulus using the same presentation format and stimuli (for both types of 
tasks). Participants were shown a black screen with 30 randomly distributed white squares. The letter ' $\mathrm{P}$ ' was displayed in 1 of the 30 squares for $500 \mathrm{~ms}$ in 1 of 433 possible font types (e.g., Times New Roman, Calibri). Participants were required to remember either the P's location or its appearance (font type). After the $\mathrm{P}$ was presented there was a $15.5 \mathrm{~s}$ delay during which participants (a) viewed a blank screen, (b) watched dynamic visual noise similar to visual white noise on a TV, or (c) performed a spatial task involving tapping a pattern on a 3 x 3 keypad. After the delay, another $\mathrm{P}$ was displayed and participants indicated whether it was the same as or different from the first $\mathrm{P}$. On trials where participants were required to remember the location of the $\mathrm{P}$, a 'same' response indicated that the $\mathrm{P}$ was displayed in the same location (the font was identical). On trials where participants were required to remember the appearance of the $\mathrm{P}$, a 'same' response indicated that the $\mathrm{P}$ was displayed in the same font (location was identical).

Darling et al. (2009), observed a double dissociation: mean response latencies were longer for location trials than for appearance trials in the tapping condition than in the visual noise condition and mean latencies were longer for appearance trials than location trials in the visual noise condition than in the tapping condition. Spatial tapping during the delay period interfered with participants' ability to remember the location of the P more than its appearance whereas watching dynamic visual noise interfered with participants' ability to remember the appearance of the $\mathrm{P}$ more than its location. This finding suggests that different components of the VSSP were required to remember an object's location versus its appearance.

Support for the idea that spatial and visual information is stored/processed by two distinct WM subsystems is provided by the results from developmental, clinical, and brain imaging studies. Vicari, Bellucci, and Carlesimo (2003) measured the visual span (appearance) and location/spatial span of a group of individuals with Williams Syndrome, a neurodevelopmental 
disorder affecting language development and motor skill acquisition. Compared to a control group, the Williams Syndrome group had the same visual span, but showed a significantly shorter location/spatial span. The results therefore indicate that Williams Syndrome selectively affects the development of the memory systems responsible for processing spatial but not visual information. This finding is supports the view that spatial and visual information are processed by independent memory components.

Logie and Pearson (1997) found developmental differences in the ability to remember spatial and visual patterns (see also Pickering et al., 2001). By using recall of visual patterns as a 'visual' memory task and recall of a pattern of movements as a 'spatial' memory task, Logie and Pearson found a pattern of divergent capacities for recognition and recall of the two types of stimuli across ages. Children ages $5-6$ showed no significant difference in their recognition and recall spans of spatial patterns and visual patterns. However, the two older age groups ( $8-9$, and $11-12)$ showed a continually increasing difference in spatial and visual spans. Although both spatial and visual spans increased with age, the recognition and recall span for visual patterns was significantly greater than for spatial patterns in the oldest group. The authors concluded that the pattern of findings suggests that there are different rates of development for memory of spatial patterns versus visual patterns and support the notion that there are two separate WM subsystems: one for visual information and one for spatial information.

Darling et al. (2006) measured visual-spatial WM in two patients with varying types of brain damage to corroborate the claim that visual and spatial information are processed by two separate WM subsystems. Patient A was a 54 year-old who suffered a stroke that damaged the temporal lobes of the right hemisphere as well as the right parietal lobe. Patient B was a 23 yearold who had suffered a traumatic brain injury causing damage bilaterally to the frontal lobes as 
well as the right parietal lobe. Patient A showed a selective deficit in memory for spatial location but not visual appearance. In contrast, patient B showed a selective deficit in memory for visual appearance, but not spatial location. If visual-spatial WM for visual appearance and spatial location were one multi-purpose store, then damage leading to deficits in one should be co-morbid with deficits to the other.

Finally, neural imaging studies have provided converging evidence that the VSSP is fractionated into visual and spatial components. Processing and rehearsal of visual information (object form and color) seems to occur more in the occipital lobe, whereas processing and rehearsal of spatial information seems to occur more in the parietal lobe (Baddeley, 1992). Furthermore, the neural location of spatial processing (not visual processing) seems to occur in the same cortical area that is activated when people are performing motor tasks, specifically movements involving spatial information (Ikkai \& Curtis, 2011). Ricciardi et al. (2006) found that when congenitally blind participants performed spatial tasks using tactile stimuli in a modified Corsi block task, the same brain region (parietal lobe) that is thought to be associated with visual-spatial WM was activated. Ricciardi et al. concluded that spatial information, regardless of modality, was processed similarly. Likewise, Fiechler, Burke, Engel, Bien, and Rosler (2008), using fMRI, also found the parietal lobe to be heavily involved in memory for spatial movements when those movements were encoded both visually and tactilely. Taken together these results demonstrate that multiple spatial tasks presented through different modalities should interfere similarly to multiple spatial tasks presented in the same modality.

In sum, visual-spatial WM is complex and potentially intertwined with sense modalities other than vision. There is converging evidence from behavioral, imaging and special population studies suggesting that visual-spatial WM is divided into two sub-components, one for handling 
spatial location (inner scribe) information and one for handling visual appearance (visual cache). The present research was conducted to take steps toward understanding how informative motion utilizes particular types of visual-spatial WM and determine if responding to motion can cause selective interference to one system (spatial or visual) but not the other.

\section{Motion Perception and Visual-Spatial WM}

For motion to be of value it must convey information regarding changes in direction or spatial location. The aforementioned research suggests that visual-spatial WM is the cognitive mechanism most involved with perceiving and processing spatial information across different modalities (visual, auditory, or proprioceptive and vestibular). Therefore, it is likely that processing multiple pieces of spatial information from any modality simultaneously should cause some amount of interference. In contrast, processing visual information and spatial information, regardless of modality, should result in less interference.

\section{Visual-Spatial WM and the Vestibular System}

Although motion in flight simulators can provide pilots with extra information that is useful in situation where information presented visually or aurally only is less salient or hard to interpret (e.g., high frequency vibrations associated with a tail rotor failure), a great deal of motion information is redundant (e.g., those associated with a velocity, direction, or altitude change) and can be largely ignored. The decision to ignore or further process motion information is likely to tax WM given that visual-spatial WM has been implicated in monitoring and responding to the visual and spatial information associated with motion. For this reason, it is important to understand the interaction between motion and WM when designing and implementing full motion flight simulators. 
In one of the few attempts to demonstrate that motion can impair WM in an applied setting, Webb et al. (2011) measured pilots' cognitive ability by administering a Paced Auditory Serial Addition Test (PASAT) in a full motion simulator under normal and disoriented flight conditions. The PASAT is a WM task believed to also index rate of information processing. In the 'normal flight' condition pilots flew with the use of full instrumentation, which lead to low reliance on aircraft motion. However, in the 'disorientating flight' condition the instruments indicating the aircraft's attitude were unavailable, which lead to a high reliance on motion. When pilots were forced to rely on motion information to maintain the aircraft's attitude, performance on the PASAT decreased compared to when pilots used the attitude indicators. Webb et al. concluded that spatial disorientation and the need to rely on motion information significantly interfered with the cognitive mechanisms required to perform the PASAT. Although these data do not conclusively show that motion selectively interferes with visual-spatial WM, they do support the idea that motion interferes with cognitive tasks that are secondary to the basic flight control tasks.

Hanes and McCollum (2006) reviewed differences in cognitive ability between patients with and without vestibular deficits to explore the possible link between visual-spatial WM and the vestibular system. Counting backwards requires a substantial amount of central executive resources (Allen, Baddeley, \& Hitch, 2014). However, Hanes and McCollum argued that because numbers are learned in order, reciting them backward also requires spatial WM to reorder the number sequence. Hanes and McCollum cite a study by Risey and Briner (1990) in which participants with vertigo counted backward by 2 and consistently skipped the tens position $(94,92,80,88,86 \ldots)$. Participants were seemingly unaware that they were making errors on the task. Hanes and McCollum suggested that instability of perceived motion (caused by faulty 
vestibular information) interferes with one's ability to spatially re-order the number sequence. People who suffer from vertigo may be continually engaged in maintaining posture, which could deplete the spatial resources required to perform the backward counting task. Likewise, pilots in motion simulators may be engaged in a sort of 'attitude first' process in which other spatial tasks are shed. Finally, Smith, Darlington, and Zheng (2010) present a review of multiple studies suggesting that peripheral vestibular lesions are highly associated with decreases in spatial WM. Thomas and Seiffert (2010) investigated the link between visual-spatial WM and selfmotion by using a multiple object tracking task. Tracking is something we use every day whether walking, driving, or sitting passively to watch a sports event. Multiple object tracking requires attending to, or shifting attention amongst, multiple targets while holding their position and movement profiles in WM (Fougnie \& Marois, 2006). Thomas and Seiffert examined the number of objects participants could track under different motion interference conditions: seated and stationary, walking in place (not moving), walking self-propelled (in a $90^{\circ}$ arc), or being passively pushed in a wheelchair (in a $90^{\circ}$ arc). Participants were shown five identical red balls through a head mounted display. Either one or three of the balls turned blue to indicate they would be the targets; the target ball(s) changed back to red after $2 \mathrm{~s}$ to match the other balls. The balls then moved around the screen for $5 \mathrm{~s}$ during which the participant was required to track the target ball(s). Participants had to indicate which ball(s) was/were the target(s). The results showed that there was no difference in accuracy across motion conditions ( $\sim 85 \%$ accuracy) when tracking one target ball. However, when tracking three target balls, accuracy suffered while walking self-propelled or being pushed in a wheelchair ( $55 \%)$ compared to sitting or walking in place $(\sim 70 \%)$. Thomas and Seiffert concluded that participants' physical movement through space (or the tracking thereof) interfered with the number of objects that could be held in spatial 
WM and tracked. Interestingly, although being pushed in a wheelchair requires no active monitoring of one's own position, tracking seems to occur automatically and requires cognitive resources.

In an attempt to quantify how much cognitive capacity is required to track one's own self-motion, Thomas and Seiffert (2011) used a similar experimental paradigm and found that the visual-spatial WM capacity required to track one's own position in space is equivalent to tracking one additional object. Simply put, tracking three objects while engaged in self-motion requires the same amount of WM capacity as tracking four objects while stationary. Thomas and Seiffert's studies $(2010 ; 2011)$ suggest that the mechanisms required to perform spatial WM tasks are also involved in the monitoring of active and passive self-motion.

In sum, although the visual system may be the most dominant mode of input for maintaining orientation (Engel et al., 2008; Fiechler et al., 2008), the vestibular system is used to orient the body in space, perceive changes in motion (direction and velocity), and adjust the body accordingly when visual stimuli are unavailable or unreliable (Gresty \& Golding, 2009; Webb et al., 2011). The process of perceiving and adjusting to changes in orientation is integral to maintaining orientation in an aircraft as well as maintaining day-to-day postural control. Postural control is the continuous adjustment of muscles and joints to remain standing upright and balanced. Postural control was once thought to be automatic (VanderVelde, Woollacott, \& Shumway-Cook, 2005), but it now seems that it is partially controlled by visual-spatial WM. If postural control draws from a limited pool of WM resources, then there should be a link between postural control and performance on concurrent cognitive tasks that require spatial processing. 


\section{Postural Sway and Working Memory}

Maintaining standing balance is a complex task that requires the online perception and integration of visual, vestibular, and proprioceptive information and subsequent manipulation of the body in response to that information (Lajoie et al., 1993; Makizako, Furuna, Ihira, \& Shimada, 2013; Maylor \& Wing, 1996). The cognitive demands associated with maintaining standing balance can be thought of as similar to those required to correct and maintain aircraft orientation. Maintaining standing balance was traditionally thought to be automatic, but this may not be the case if spatial WM is required to maintain or regain standing orientation. If something as easy as maintaining postural control requires cognitive resources, then there is reason to believe that even more cognitive resources would be required when perceiving and responding to more complex motion such as those produced by simulated (or real-world) aircraft.

Standing requires constant updating of one's position in space and making necessary corrections (Riley et al., 2012). Postural sway can be recorded by measuring body displacement in the lateral and posterior/anterior directions. Postural sway is a useful measure because it can be made more difficult by changing standing position to something less natural and more challenging and shows clear interference when paired with some cognitive tasks (VanderVelde et al., 2005). Postural sway is increased when participants perform spatial memory or executive function tasks (Makizako et al., 2013; Woollacott \& VanderVelde, 2008).

Lajoie et al. (1993) used a simple reaction time task to assess the attentional demands associated with sitting, standing, and walking. They found that even simple reaction times to auditory stimuli increased as the participants moved from sitting to standing and then to walking. They attributed this increase in reaction time to the increase in cognitive resources required to maintain balance. This study, however, did not examine which cognitive resource(s) is/are being 
used in these various tasks. To further investigate which WM components were involved with postural control, Maylor and Wing (1996) had participants perform tasks that taxed specific WM subsystems and measured their effects on postural sway. Participants randomly generated numbers to load the central executive, counted backwards to load the VSSP (possibly by activating spatial processing of the number sequence; see Hanes and McCollum, 2006), or counted forwards to load the articulatory loop. The results showed that backward counting and random number generation increased postural sway. Maylor and Wing suggested that because tasks that require central executive or spatial WM capacity interfere with the processing of visual and vestibular information (to maintain balance), they likely both use the same cognitive resources.

Building on the assumption that the VSSP is the primary WM subsystem responsible for postural control, and that visual-spatial WM is likely separated into visual and spatial systems, VanderVelde et al. (2005) investigated which of these further divisions of visual-spatial WM was more involved in postural control. VanderVelde et al. displayed a load figure (to be remembered) for $2 \mathrm{~s}$, a blank screen for $3 \mathrm{~s}$, and a probe item (to be compared to the load figure) for $1 \mathrm{~s}$, at which point participants had to verbally respond 'yes' if the probe was the same as the load figure or 'no' if it was different. Participants were required to either remember the load figure's appearance or its location on the screen. During this task, participants were either seated, standing in a comfortable stance with feet side-by-side, or standing with one foot placed in front of the other. Accuracy scores on the location WM task were significantly lower in the increased complexity stance condition than in the seated or comfortable stance conditions. This difference was not observed for the appearance WM task. VanderVelde et al. therefore concluded that 
spatial WM, rather than visual WM, was most affected by maintaining unstable stances (see also Makizako et al., 2013; Ramenzoni et al. ,2007).

Although VanderVelde et al. (2005) attempted to divide visual from spatial WM, a potential confound exists in that the stimuli in both the visual and spatial conditions were presented visually. Granted, most spatial information is closely tied to visual information in the real world, but if there is a link between postural sway and spatial WM, then a purely spatial task should have an effect on postural sway. To address this possible confound, Woollacott and VanderVelde (2008) replaced the visual-spatial WM task with non-visual, spatial WM tasks. Memory tasks were performed while participants remained seated, stood with feet shoulder width apart (easy stance), or stood in a more difficult-to-maintain stance with their feet close together. Participants were further required to perform a visual memory task, an auditory tone memory task, or an auditory spatial memory task. The visual memory task was an $n$-back task in which pictures of random objects were presented visually once per second. If the current object was the same as the object presented $n$ pictures back, then participants responded by saying 'yes'. The auditory tone memory $n$-back task was identical to the object memory task except that tones were presented instead of pictures. The auditory spatial memory $n$-back task consisted of tones played from 8 speakers placed equidistant from each other around the room. Tones were played once per second from one randomly selected speaker. If the current tone was from the same speaker as $n$ tones back, then participants responded by saying 'yes'.

There was no difference in response latencies on the object/tone memory tasks presented aurally and visually when sitting or standing. However, response latencies on the auditory spatial memory task were significantly longer in the standing condition than in the sitting condition. Furthermore, postural sway while standing was greatest when performing the auditory spatial 
memory task. These results suggest that spatial tasks, regardless of whether they are presented aurally (Woollacott \& VanderVelde, 2008) or visually (VanderVelde et al, 2005), interfere with postural control.

Riley et al. (2011) found that varying the presence or absence of a reference frame in which a spatial WM task was presented had an affect on postural sway. A reference frame can be defined as the stable, unchanging, frame that encloses specific stimuli. For example, the black frame of a computer monitor could be viewed as a reference frame. Stimuli presented on a computer screen cannot move outside that frame and thus are anchored to a constant and finite area. Increasing the size of the computer monitor to a large wall size monitor while leaving stimulus size unchanged increases the frame of reference. Remembering the location of a stimulus on a small frame of reference is easier than remembering the location of an equally sized stimulus on a large frame of reference because it increases the amount of possible error by increasing the possible distance between the stimuli and the nearest part of the reference frame. Riley et al. (2011) reported that postural sway decreased if spatial WM tasks were presented in a small, defined reference frame. If the WM tasks were presented in ill-defined reference frames where the background (unrelated to the task) could be mistaken for part of the reference frame, then postural sway increased. In short, varying the reference frame can increase or decrease the difficulty of the WM task, and can impact ones' ability to maintain balance. It seems as though there is some overlap between spatial WM and the proprioceptive and vestibular processing required maintain postural control.

In sum, there is convincing evidence that the ability to maintain postural control and orientation is susceptible to interference caused by concurrent tasks that tax spatial WM. Given that perceiving and responding to external motion is a more complex than maintaining postural 
control, it is reasonable to assume that loading spatial WM will have interfere with tasks that require the observer to process external motion, and vice versa.

\section{Effects of Eye and Limb Movements on Spatial Working Memory}

The dependency of postural control on spatial WM extends to the perception, planning and execution of limb movements (Goble et al., 2012) and eye movements (Lawrence, Myerson, \& Abrams, 2004; Lawrence et al., 2001; Postle et al., 2006; Theeuwes, Belopolsky, \& Olivers, 2009). Indeed, eye and limb movements may interfere with spatial WM or may require spatial WM for normal operation. Knowing where your limbs are in space without looking at them or being able to place them in a remembered position without watching them move is a valuable asset. For example, pilots focusing on a distant runway do not have to look down at their hands to see the position of the aircraft's controls. Proprioceptive feedback from muscles and joints allow pilots to maintain memory of their limb position. Patients with Cerebral Palsy (CP), however, often suffer from a reduced sense of proprioception (Goble et al., 2012), which makes it difficult to locate their limbs in space, or to remember the angle at which a joint has been bent, without looking at them. Goble et al. measured CP patients' ability to remember arm bend angle using an Ipsilateral Remembered task (IR) in which an arm is bent to a measured angle, then after a delay period ( 2 or $15 \mathrm{~s}$ ), the participant is asked to put their arm in the original position and the angle error is measured. Expectedly, CP patients performed quite poorly. However, the authors also found that performance on the IR task correlated with scores on a spatial memory task (Corsi block-tapping task).

Goble et al. (2012) found that participants whose accuracy on the IR task improved when given a longer time to encode the arm-bend-angel had higher initial spatial WM scores than participants who did not benefit from a longer encoding time. Increasing the encoding time of a 
WM task should improve performance on that task. Goble et al. suggested that the IR task is a spatial WM task and participants with a high spatial WM ability were capable of recruiting their spatial WM to improve IR task accuracy when given extra encoding time, thus improving performance. However, participants with a low spatial WM ability had no means of improving their performance on the IR task even with more time to encode. These findings provide clear evidence that spatial WM supports the systems/processes involved in proprioception (as seen in $\mathrm{CP}$ patients). It therefore follows that any tasks that require cognitive resources to monitor proprioception (e.g., responding to motion cues) could interfere with concurrent spatial tasks.

Lawrence et al. (2001) examined the extent to which reflexive saccades, pro-saccades, and anti-saccades interfere with memory of spatial locations versus memory of letter spans. Reflexive saccades occur after shifts of the eye to a peripheral stimulus or after the disappearance of a fixated stimulus, whereas anti-saccades occur when movement towards a stimulus is inhibited, and pro-saccades are associated with planned eye movements. Lawrence et al. used a dual-task paradigm in which participants completed either a spatial memory task or a verbal memory task with an intervening eye movement task. The spatial memory task required participants to memorize the locations of a series of Xs presented throughout a $4 \times 4$ grid and later indicate their locations. The verbal memory task required participants to remember a string of letters presented on a screen in serial order. The induction of saccades (largely reflexive saccades) interfered with the spatial memory task but not the verbal memory task. However, because eye movements can also lead to shifts of visual attention, the interference observed on the spatial WM task may be due to attentional shifts and not to motor movements of the eye.

To address the possibility that interference on spatial tasks due to eye movements could be due to a shift in attention, Lawrence et al. (2001) conducted an experiment that used the same 
WM tasks and measured the interference caused by simple fixation, reflexive saccades, and left/right arm movements. Limb movements and reflexive saccades produced the same level of interference on spatial but not on verbal WM. However, although movement significantly interfered with spatial WM, shifts of spatial attention may also interfere to some degree. In a follow-up study controlling more heavily for spatial attention, Lawrence, Myerson, and Abrams, (2004) found that both shifts of attention and eye movements independently interfered with spatial WM. In sum interference on WM caused by saccadic eye movements is likely not related to shifts of visual attention.

Postle et al. (2006) replicated four unpublished studies that were previously conducted by Baddeley, which investigated the effects of eye movements on WM. Postle et al. found that eye movements interfered with visual-spatial WM but not verbal WM; eye movements interfered with memory for the location of objects (spatial memory) but not the visual components (shape/color); eye movements produced the most interference while viewers maintained information in WM rather than encoded; and lastly, the control of eye movements, more than the eye movements themselves, were most disruptive to spatial WM. These findings suggest that other types of body movements that require a of control are more disruptive to WM than those that occur involuntarily or automatically.

When motion is produced by something other than the body itself (e.g., in simulated motion environments), the control of motor movements (limb or ocular) should become increasingly more complex or even more difficult. For example, scanning an approach map before flight (in a stationary environment) requires only pro-saccades because there are no external forces on the body due to movement. However, in a moving environment (e.g., during flight), every movement made by the individual has to be controlled based on the planned 
movements plus any forces exerted on the body by the moving environment, this adds a layer of complexity to controlling limb and body movement. If spatial WM is required to store and process movement-related information, then interference should be observed in a dual-task study involving spatial WM and motion.

\section{Simulated Motion and Spatial Working Memory}

Active self-motion is different from passive self-motion (e.g., walking vs. pushed in a wheelchair) in that the former allows crucial information regarding the direction and magnitude of motion to be collected through the action of muscle and joint movements, whereas the latter results from proprioceptive information generated by external forces. What happens if people are required to monitor passive movement through space rather than monitoring their own selfpropelled movements? In Yardley et al. (1999), participants sat in a rotating chair in a darkened room devoid of visual information. They monitored how far they had been rotated based on vestibular information and responded by using a joystick to rotate themselves back to the point of origin (e.g., if they were rotated $60^{\circ}$ clockwise, the correct response would be to rotate themselves $60^{\circ}$ counterclockwise). While being rotated, participants were instructed to (a) focus only on the rotation, (b) respond to auditory stimuli as fast as they can while monitoring their rotation, or (c) count backward and monitor their rotation. There was also a control condition in which participants were rotated, but did not re-orient themselves and therefore did not have to actively monitor the rotation. Participants also completed each of the WM tasks in isolation to establish a baseline for each participant. Yardley et al. found that rotation, regardless of whether participants had to monitor it or not, resulted in increased response times to the auditory stimuli. However, responding to the auditory stimuli did not affect participants' ability to return the seat to its starting position. Backward counting while monitoring rotational motion impaired the 
ability to return the seat to its origin. These findings show that merely monitoring changes in orientation induced by external forces (passive motion) requires measureable cognitive processing. Yardley et al. suggest that the visual-spatial demands of backwards counting can interfere with one's ability to accurately monitor motion.

Vestibular motion typically occurs in conjunction with visual information in real-world environments, so Yardley et al.'s (1999) findings demonstrate interference between vestibular information in the absence of vision and may not generally examine the combined effects of vestibular and visual motion information. Gresty et al. (2003) had participants sit in a full-motion flight simulator with a horizon presented visually on the monitors. Participants performed a Brooks matrix task to load spatial WM, and a Brooks verbal task to load verbal WM. The simulator (a) remained stationary, (b) oscillated by pitching up and down with visuals matching the motion produced by the pitching of the simulator, or (c) pitched up and down with inverse, mismatched visuals while participants performed the WM tasks. Gresty et al. (2003) found that error rates were not significantly different on either the spatial or verbal tasks in either the stationary or moving condition with matched visuals. However, when participants were oscillated in the mismatched visual condition (disoriented) there was a significant increase in errors on the spatial memory task, but not on the verbal memory task. Motion sickness does not appear to be the reason for these results as performance on the verbal task did not suffer. Gresty et al. suggest that matching visual and vestibular motion can be easily ignored, however, inconsistent motion information leads to an automatic attempt to devote spatial resources to monitoring the motion. Thus, when spatial information presented in two modalities is inconsistent more spatial resources are consumed. 
Based on Gresty et al.'s (2003) findings, it appears that adding visual information reduced the interference of passive motion on concurrent WM tasks. This effect may have occurred because the visual system dominates in the perception of motion. Thus when visual information is present, vestibular information is weighted less heavily, which frees up the cognitive resources required to perform a secondary spatial task. However, a mismatch between visual and vestibular information produces selective interference on spatial tasks, even though there was no requirement to respond to or even monitor the motion. These findings suggest that similar to Thomas and Seiffert's $(2010 ; 2011)$ findings, participants automatically monitor vestibular and proprioceptive motion and this monitoring requires WM capacity

\section{Summary}

People use vestibular and proprioceptive information to monitor spatial position or orientation. The aforementioned research demonstrates that postural control, active, and passive (self-) motion all require people to monitor and integrate visual, vestibular, and proprioceptive information and that these activities require visual-spatial WM. However, motion processing seems to selectively interfere with tasks that utilize spatial WM while leaving visual WM unaffected. This pattern of results suggests that spatial information and visual information may be processed in separable components of visual-spatial WM.

\section{Present Research}

The objective of the present research was to further examine the link between visualspatial WM and the perception of motion. Based on the multi-component model of WM, and the aforementioned research, it is suggested that motion perception primarily utilizes the spatial component of visual-spatial WM. Therefore, it is hypothesized that memory tasks requiring spatial WM will be impaired by completion of a concurrent motion cue discrimination task, 
whereas memory tasks requiring visual WM will not be impaired. Two experiments were conducted in which visual and spatial WM tasks were paired with a motion cueing task. Experiment 1 investigated interference caused by responding to motion cues during rehearsal of visual stimuli. Experiment 2 investigated interference caused by responding to motion cues during encoding of visual stimuli.

\section{Experiment 1}

Experiment 1 examined how the processing of motion cues interferes with the retention of visual (appearance) information versus spatial (location) information. Working memory tasks were developed to measure participants' memory for either spatial information or visual information using the same stimuli. Experiment la was designed to equate the difficulty of the two variants of the WM task. In Experiments $1 \mathrm{~b}$ and $1 \mathrm{c}$, participants were required to remember either the location or the appearance of the stimuli. On half of the trials, participants were required to discriminate between left/right motion cues during a $15 \mathrm{~s}$ retention interval. It was hypothesized that the motion discrimination task would decrease performance on the spatial memory task but not on the visual memory task. In Experiment $1 \mathrm{~b}$ three motion cues were used whereas in Experiment 1c ten motion cues were used. To briefly preview the results, the motion cue discrimination task significantly impaired memory for spatial information, but not for visual information.

\section{Experiment 1a}

The purpose of Experiment 1a was to equate the difficulty of the spatial WM task and visual WM task developed for Experiment 1. To this end, performance on these two WM tasks was measured without any motion cueing. 


\section{Method}

\section{Participants}

Ten graduate students volunteered to participate. Ages ranged from 23 to 38 years $(M=$ 26.67, $S D=4.66)$. All participants were right handed. No participants reported any history of back injury, concussion, or head injuries and all were assumed to have normal or corrected-tonormal visual acuity.

\section{Design}

A one factor (Memory Task: Spatial vs. Visual) repeated measures design was used. Memory Task was blocked and counterbalanced across participants.

\section{Stimulus Materials and Presentation}

A modified version of Darling et al.’s (2007) “Letter P" task, in which participants were instructed to either remember the location or the appearance (font type) of letter Ps presented on a screen, was developed for the current experiment. Location was manipulated by consecutively presenting two target Ps (for $500 \mathrm{~ms}$ each) in black ink on a white background in two different randomly selected cells of an invisible 4 x 6 grid. The 4 x 6 grid was not displayed, thus the target Ps seemed to appear in random locations on the screen. Following a retention interval, two probe Ps were simultaneously presented. On "same" trials, the probe Ps appeared in the same cells as the target Ps. On "different" trials, one of the probe Ps moved to an adjacent cell. The number of candidate cells that a probe $\mathrm{P}$ could move to varied from eight to three, depending on whether its corresponding target $\mathrm{P}$ was centered in the grid, along an edge, or in a corner. The appearance of the Ps was manipulated by selecting 48 font types ( 24 serif, 24 sans-serif) from Microsoft Office's (2008) list of fonts. The two groups of 24 font types were sub-divided into 12 typical and 12 atypical fonts based on feedback from a sample $(n=10)$ of naïve individuals. The 
individuals were asked to sort the serif and sans-serif groups into 12 "typical" and 12 “atypical" fonts each (see Figure 4 for examples). The average agreement between individuals on which group a particular font type should belong to was $90 \%$. Dividing the Ps into four groups (serif/sans-serif $\mathrm{x}$ typical/atypical) provided an objective method of rating the Ps in terms of visual similarity. In order to control the degree of change between a target and probe $\mathrm{P}$ on a "different" trial, the Ps were only allowed to differ along one category dimension. For example, if one of the target Ps was sans-serif and typical, then the 'different' probe $\mathrm{P}$ had to either be sans-serif and atypical or serif and typical. In other words, a P could not be replaced with another $\mathrm{P}$ that came from the same category dimensions, nor could it be replaced by a $\mathrm{P}$ that differed on both category dimensions. All Ps subtended a visual angle of approximately $4.8^{\circ}$ both vertically and horizontally.

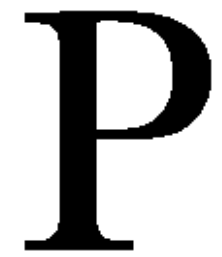

Typical Serif

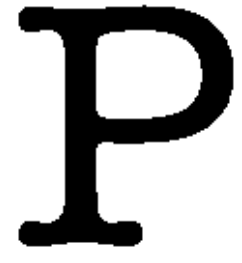

Atypical Serif

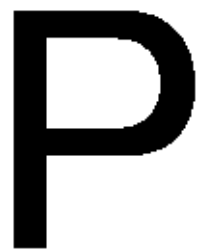

Typical Sans Serif

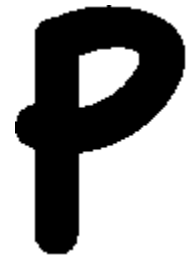

Atypical Sans Serif

Figure 3. Examples of a "P" from each of the four category dimensions used as stimuli in experiments $1 \mathrm{a}, 1 \mathrm{~b}$, and $1 \mathrm{c}$.

\section{Apparatus}

Stimuli were presented on a 42" Barco monitor running at $60 \mathrm{~Hz}$ with a screen resolution of 1920 x 1080 . The monitor was stationary and located approximately $120 \mathrm{~cm}$ in font of participants with the midline of the monitor at eye height. The program responsible for counterbalancing order, stimulus presentation, and data logging was programmed in $\mathrm{C \#}$. Participants responded using a Cedrus Model RB-530 serial response pad, which accurately 
records responses to the nearest millisecond. Participants pressed up/down to indicate a same/different response to the letter P task.

\section{Procedure}

Participants were tested in a quiet, distraction-free room. Following the completion of an informed consent form (Appendix A) and a basic demographic questionnaire, participants were seated in a motion cueing seat (even though no motion cues were provided), which was adjusted for height to ensure that their feet rested comfortably on a platform. The seat was then moved forward/backwards and the monitor was moved up/down to hold viewing distance and angle constant across participants.

Participants completed a practice session that consisted of 4 Spatial trials and 4 Visual trials, with two same/different trials in each condition. On the Visual trials, the letter P's font type was manipulated. On the Spatial trials, the letter P's location was manipulated. Two experimental blocks followed the practice session. One block consisted of 30 Spatial trials and the other consisted of 30 Visual trials with block order counterbalanced across participants. Each block lasted approximately 11 minutes with a five-minute break between blocks.

Prior to each experimental block, participants were instructed to remember either the location (Spatial condition) or the appearance (Visual condition) of the two consecutively presented target Ps. Each trial began with the presentation of a blank white screen for $1 \mathrm{~s}$, followed by the first target $\mathrm{P}(\mathrm{P} 1)$, chosen at random from the 48 fonts in one of 24 possible screen locations for $500 \mathrm{~ms}$. The second target P (P2) was then displayed for $500 \mathrm{~ms}$ in one of the remaining 23 locations on the screen. Static visual noise that filled the entire monitor was then presented for 15 seconds. This was followed by a 3 -second presentation of the two probe Ps. 
Participants were required to indicate whether the target and probe Ps were the same or different as accurately as possible.

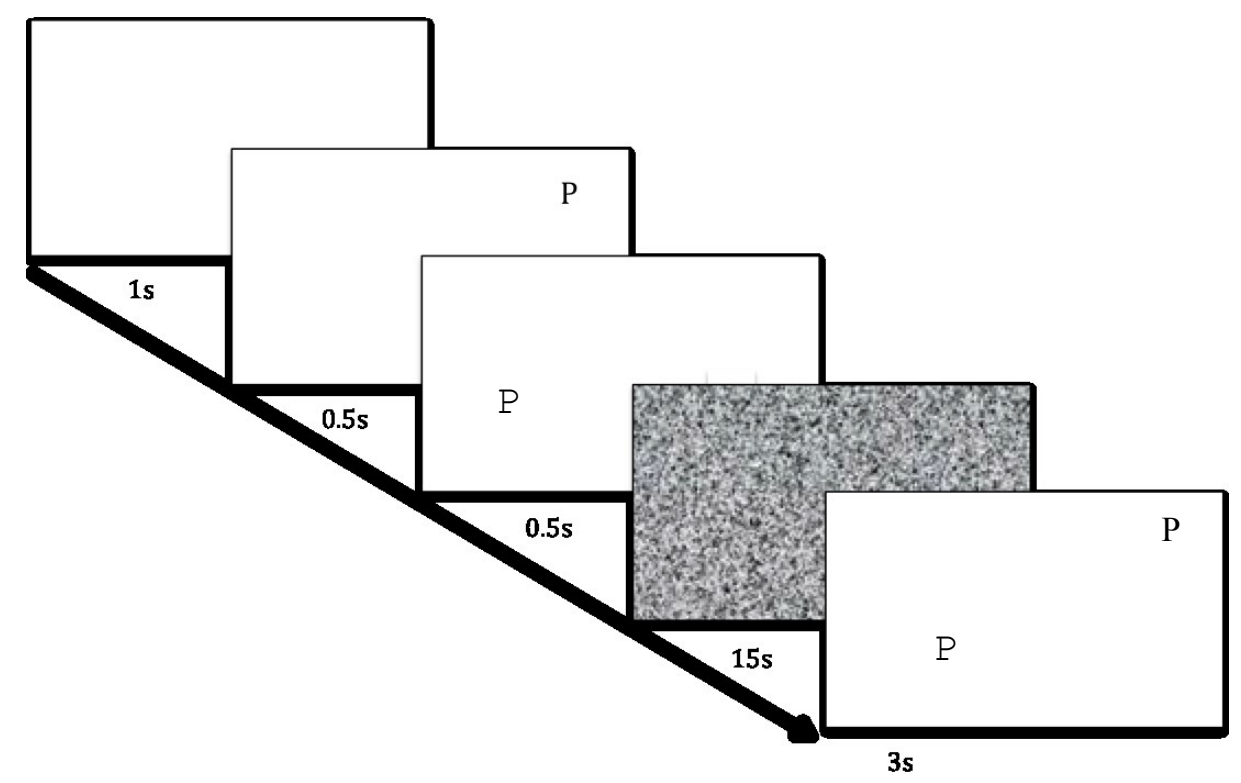

Figure 4. Example of a "same" spatial or visual trial sequence from Experiments 1a, 1b, and 1c.

\section{Results and Discussion}

Experiment 1a was designed to determine whether the Visual and Spatial conditions of the letter P task were equally difficult and to ensure that performance on this task would not be subject to floor or ceiling effects. Responses to the memory trials were recorded as correct or incorrect. Accuracy was calculated as the number of correct responses divided by the total number of trials.

A one-way repeated-measures ANOVA revealed that there was no difference in accuracy between the Spatial $(M=82 \%, S D=38 \%)$ and Visual $(M=82 \%, S D=39 \%)$ conditions $(F<1)$. It was therefore concluded that the Spatial and Visual conditions were equally difficult. Further, 
given that the accuracy scores were above chance (50\%) and below 100\%, there was no reason to believe that using these parameters in later experiments would produce ceiling or floor effects.

\section{Experiment 1b}

Experiment $1 \mathrm{~b}$ examined whether discrimination between motion cues interferes with the rehearsal of spatial (location) information and/or visual (appearance) information. It was hypothesized that responding to motion cues would selectively interfere with the Spatial condition. The same visual and spatial WM tasks from Experiment 1a were used. Participants were required to discriminate between 3 directional (left and right) motion cues during the $15 \mathrm{~s}$ retention interval of the memory trial.

\section{Method}

\section{Participants}

Twelve undergraduate students were recruited using Carleton University's online sign-up system and participated in exchange for $1.0 \%$ course credit. Ages ranged from 17 to 28 years $(M$ $=20.1, S D=3.2$ ). Eleven participants were right handed and one participant was left-handed. No participants reported any history of back injury, concussion, or head injuries and all were assumed to have normal or corrected-to-normal visual acuity.

\section{Design}

A 2 (Motion Cueing: Motion vs. No Motion) x 2 (Memory Task: Spatial vs. Visual) repeated measures design was used. Both Motion Cueing and Memory Task were blocked factors, with presentation order counterbalanced across participants using a Latin-square design.

\section{Stimulus Materials and Presentation}

The same letter P task used in Experiment 1a was used here. 


\section{Apparatus}

The same serial response pad used in Experiment 1a was used, except that participants now pressed the left/right buttons to indicate the direction of the motion cue in addition to pressing the up/down buttons to indicate a same/different response to the letter P task. The motion cues were provided by a Rotary Aircraft True $\mathrm{Q}^{\mathrm{TM}}$ Dynamic Motion Seat from ACME World Wide Enterprises Inc., capable of simulating pitch, roll, and yaw forces. Movements were restricted to roll forces in order to provide unambiguous left/right motion cues. Motion cues were administered by simultaneously dropping the seat pan approximately $1.3 \mathrm{~cm}$ and laterally displacing the back pad approximately $1.3 \mathrm{~cm}$ in the desired direction. The full range of motion for the seat pan and back pad was approximately $2.5 \mathrm{~cm}$. The duration of the seat pan and back pad cues was roughly $100 \mathrm{~ms}$ and were executed at a rate that did not exceed $200 \%$ s. The seat pan and back pad returned to level following the cue. Although the motion seat and the letter $\mathrm{P}$ task were controlled by separate computers, the commands to display the visual stimuli and to execute a motion cue were produced by the same program. This resulted in a brief (less than 80 $\mathrm{ms}$ ), but constant, lag between the command to move the seat and the execution of the motion cue.

\section{Procedure}

The experimental set-up was identical to Experiment 1a. Once seated in the motion seat, participants completed a practice session, a motion control condition, and the four experimental conditions created by crossing Motion Cueing (Motion vs. No Motion) and Memory Task (Spatial vs. Visual). The practice session was identical to the practice session in Experiment 1a. The motion control condition required participants to respond left/right to 30 motion cues as fast as they could without a memory task. Motion cues were presented every 1.5 to $5 \mathrm{~s}$ (randomly 
determined). Participants then completed the four experimental conditions, each of which consisted of 30 trials. The four conditions were blocked and counterbalanced across participants such that each possible condition order was tested. Each block lasted approximately 11 minutes with five-minute breaks between blocks.

Prior to each experimental block, participants were instructed to remember either the location or the visual appearance (font type) of the two Ps that would be presented. The trials with motion cues were identical to those without motion, except that 3 cues were presented during the $15 \mathrm{~s}$ retention interval. The first motion cue was presented at least 1 second following the onset of the retention interval and the third motion cue was presented at least 1 second prior to the presentation of the probe display. The second motion cue was presented at a random interval no more than $1.5 \mathrm{~s}$ after or before the $1^{\text {st }}$ and $3^{\text {rd }}$ cue respectively. Participants were instructed to respond as quickly as possible to the three motion cues by pressing the appropriate button(s) on the response pad.

\section{Results and Discussion}

Data for four measures of interest were collected: motion cue response time and accuracy and response time and accuracy on the spatial and visual WM tasks. The results from the analysis of each of these four measures of performance are presented in the following four subsections.

\section{Motion Cue Response Time}

Response time was measured as the difference (in ms) between the initiation of the seat's motion and the participant's button press response. Each participant responded to 210 motion cues in total. Thirty motion cues were presented in isolation in the motion control condition to establish baseline performance. The remaining 180 motion cues occurred in the 30 spatial and 30 
visual trials in the "Motion" condition, with three motion cues being presented on each trial.

Only correct responses to the motion cues were included in the response time analysis. The data were submitted to a one-factor (Memory Task: Baseline (no WM task) vs. Spatial vs. Visual) repeated measures analysis of variance (ANOVA). The effect of Memory Task was not significant, with similar response times in the Baseline $(M=391 \mathrm{~ms}, S D=62 \mathrm{~ms})$, Spatial $(M=$ $405 \mathrm{~ms}, S D=61 \mathrm{~ms})$ and Visual $(M=395 \mathrm{~ms}, S D=52 \mathrm{~ms})$ conditions $(F<1)$.

\section{Motion Cue Accuracy}

Accuracy was recorded as a binary (correct vs. incorrect) response to each motion cue. These data were submitted to the same repeated measures ANOVA used in the motion cue response time analysis. Accuracy varied across the memory task conditions, $F(2,22)=3.24$, MSE $.001, p=.058, \eta^{2}=.228$. As seen in Figure 5, planned comparisons based on $95 \%{ }^{1}$ confidence intervals show that participants were more accurate responding to cues in the Baseline condition $(M=97.1 \% S D=3.6 \%)$ than the Spatial condition $(M=93.6 \%, S D=4.6 \%)$. Accuracy in the Visual condition $(M=95.0 \%, S D=3.6 \%)$ did not differ from the other two conditions.

${ }^{1}$ All confidence intervals in this thesis were calculated using the MSE from the corresponding ANOVA based on the statistical procedure developed by Loftus and Masson (1994) and Jarmasz and Hollands (2009). Means that are significantly different are those with confidence intervals overlapping by less than half the distance between the interval end and the interval mean. 


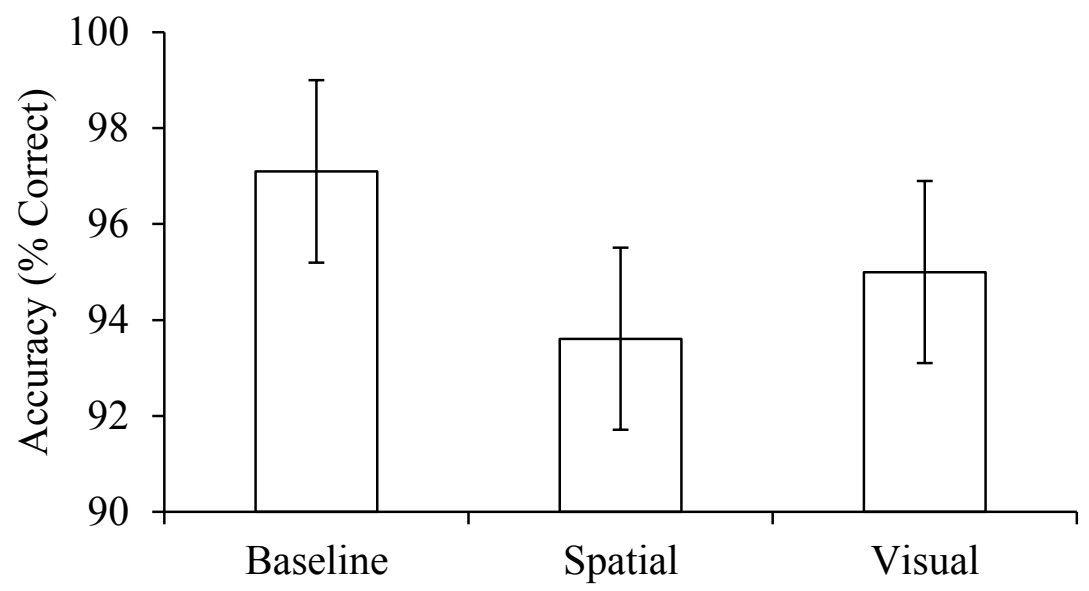

Figure 5. Mean accuracy on the motion cue task as a function of Memory Task in Experiment 1b. Error bars are $95 \%$ confidence intervals.

\section{Memory Task Response Time}

Response times were measured (in ms) from the onset of the probe display (simultaneous presentation of two Ps) until a same/different response button was pressed. Correct response times were submitted to a 2 (Memory Task: Spatial vs. Visual) x 2 (Motion Cueing: Motion vs. No Motion) repeated measures ANOVA. There was a significant main effect of Memory Task, $F$ $(1,11)=20.05, M S E=34543.966, p=.001, \eta^{2}=.646$, with response times being longer in the Spatial condition $(M=1451 \mathrm{~ms}, S D=236 \mathrm{~ms})$ than in the Visual condition $(M=1211 \mathrm{~ms}, S D=$ $254 \mathrm{~ms})$. There was neither a main effect of Motion Cueing nor a Memory Task by Motion Cueing interaction $\left(F_{\mathbf{s}}<1\right.$; see Figure 6$)$. 


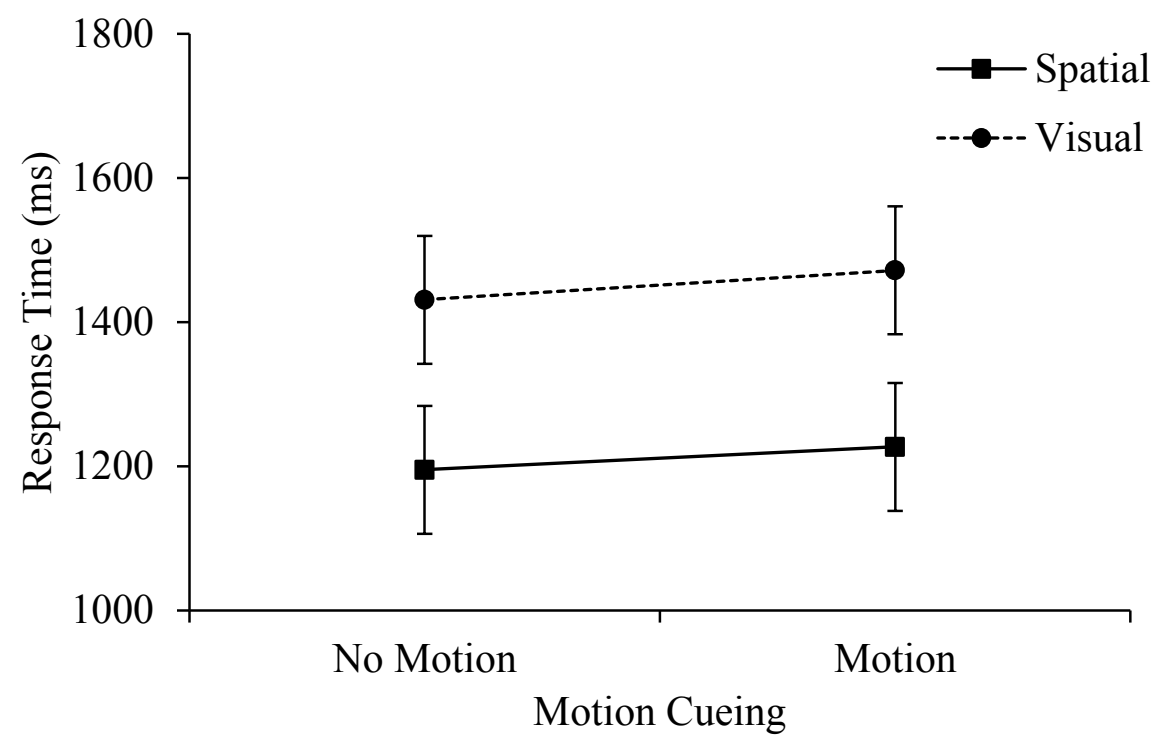

Figure 6. Mean response time (in ms) on the memory task as a function of Motion Cueing and Memory Task in Experiment 1b. Error bars are 95\% confidence intervals.

\section{Memory Task Accuracy}

Accuracy was recorded as a binary (correct vs. incorrect) response on each memory trial. The accuracy data were submitted to the same ANOVA used to analyze the memory task response time data. The main effects of Memory Task and Motion Cueing were not significant $(F \mathrm{~s}<1)$. The interaction was not significant, $F(1,11)=3.33, M S E=.007, p=.095, \eta^{2}=.232$. Figure 7 shows that mean accuracy on the spatial WM task is higher $(M=81.3 \%, S D=9.1 \%)$ than on the visual WM $(M=77.2 \%, S D=11.0 \%)$ task when no motion cues are presented. However, when participants are required to respond to motion cues, this pattern reverses and mean accuracy on the visual WM task is higher $(M=83.4 \%, S D=17.2 \%)$ than on the spatial WM task $(M=78.5 \%, S D=8.1 \%)$. 


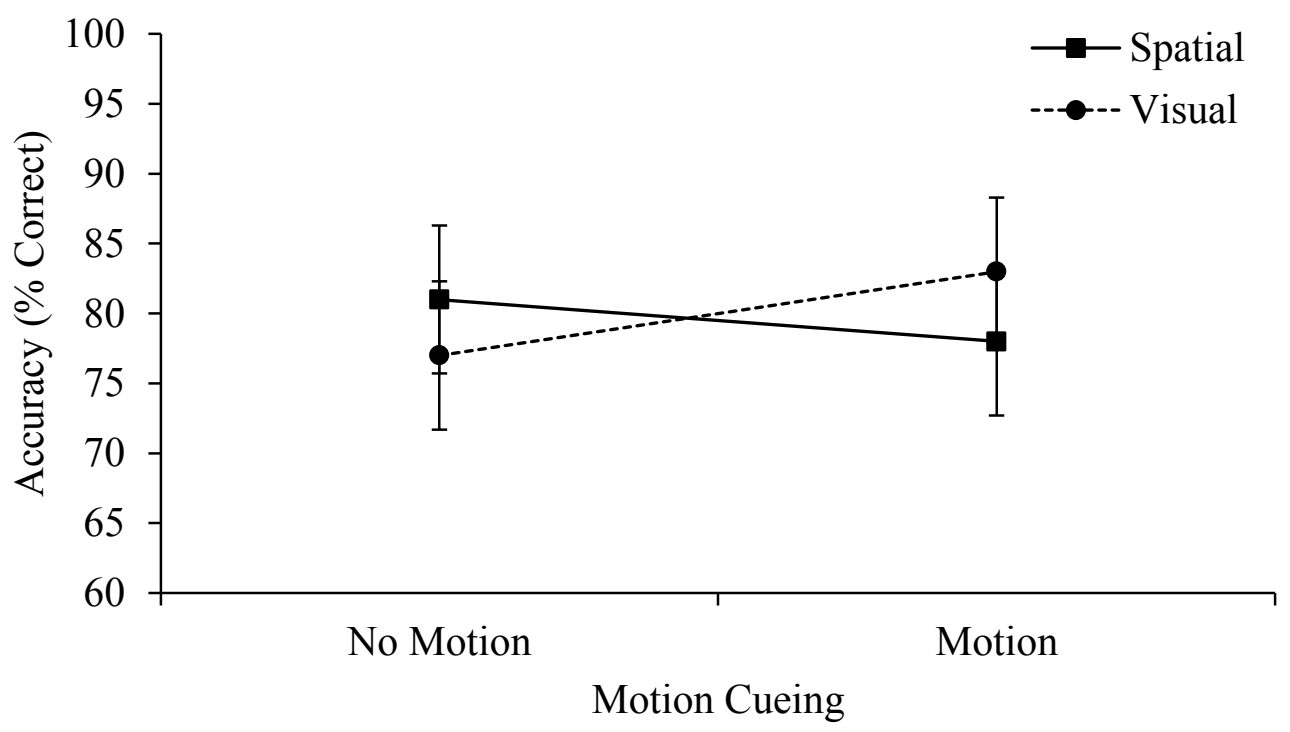

Figure 7. Mean accuracy on the memory task as a function of Motion Cueing and Memory in Experiment 1b. Error bars are 95\% confidence intervals.

\section{Summary}

In summary, while there was no effect of Memory Task on motion cue response times, the spatial WM task-relative to the baseline and visual tasks-interfered with motion cue response accuracy. Although there was some evidence that a spatial WM task interfered with discriminating the direction of motion cues, this effect was not bi-directional; the presence of motion did not interfere with WM, as evidenced by the null effects of Motion Cueing in the response time and accuracy data. Although not significant, the means of Motion Cueing and Memory Task interaction crossover and motion appears to impair performance on the spatial WM task. One possible explanation for the lack of strong and consistent evidence that processing motion interferes with (working) memory tasks is that the motion cue discrimination task was not difficult enough to tax WM. This possibility is supported by near-ceiling accuracy on the motion cue discrimination task as well as the finding that there were no response time differences on the motion cue task between the baseline condition (no WM task) and the 
Spatial/Visual conditions in which a concurrent WM task was performed. It was therefore decided that the difficulty of the motion cueing task should be increased. To this end, the number of motion cues that occurred during the $15 \mathrm{~s}$ retention intervals was increased from three to ten in Experiment 1c.

\section{Experiment 1c}

Experiment $1 \mathrm{c}$ was designed similarly to Experiment $1 \mathrm{~b}$, however, the number of motion cues was increased from 3 to 10 to increase the difficulty of the motion cueing task.

\section{Method}

\section{Participants}

Twenty undergraduate students were recruited using Carleton University's online sign-up system and participated in exchange for $1.0 \%$ course credit. Ages ranged from 17 to 31 years $(M$ $=20.3, S D=3.0)$. All participants were right handed. No participants reported any history of back injury, concussion, or head injuries and all were assumed to have normal or corrected-tonormal visual acuity.

\section{Design}

The same experimental design, stimulus material, and apparatus used in Experiment $1 \mathrm{~b}$ were used here.

\section{Procedure}

The procedure was identical to Experiment $1 \mathrm{~b}$ except that the number of motion cues during the $15 \mathrm{~s}$ retention intervals was increased from three to ten. On each of the 60 trials in the Motion Cueing condition (30 Spatial trials and 30 Visual trials), ten motion cues were presented for a total of 600 motion cues. In order to increase the number of motion cues while holding the duration of the retention interval constant, cues were randomly presented with a minimum inter- 
cue interval of $500 \mathrm{~ms}$ and a maximum inter-cue interval of $9500 \mathrm{~ms}$ with an average inter-cue interval of $1555 \mathrm{~ms}$. There was also a minimum of $500 \mathrm{~ms}$ between the beginning of the delay period and the first cue, and between the last cue and the presentation of the probe display. The timing of the cues in the motion control condition was adjusted to mirror the presentation rate of the cues in the Spatial/Visual conditions.

\section{Results and Discussion}

The same four measures of interest in Experiment $1 \mathrm{~b}$ are reported in the following subsections.

\section{Motion Cue Response Time}

Response time was measured as the difference (in $\mathrm{ms}$ ) between the initiation of the seat's motion and the participant's button press response. Each participant responded to 630 motion cues in total. Thirty motion cues were presented in isolation in the motion control condition to establish baseline performance. The remaining 600 motion cues occurred in the 30 spatial and 30 visual trials, with ten motion cues being presented on each trial. Only correct responses to the motion cues were included in the response time analysis. The data were submitted to a one-factor (Memory Task: Baseline (no WM task) vs. Spatial vs. Visual) repeated measures ANOVA. The effect of Memory Task was not significant, $F(2,38)=2.30, M S E=1652.402, p=.115, \eta^{2}=$ .108.

\section{Motion Cue Accuracy}

Accuracy was recorded as a binary (correct vs. incorrect) response to each motion cue. These data were submitted to the same repeated measures ANOVA used in the motion cue response time analysis. The effect of Memory task was not significant $(F<1)$, with no statistical 
differences in accuracy between the Baseline $(M=90.1 \%, S D=22.1 \%)$, Spatial $(M=91.5 \%$, $S D=12.2 \%)$, and Visual $(M=91.8 \%, S D=12.6 \%)$ conditions

\section{Memory Task Response Time}

Response times were measured (in ms) from the onset of the probe display (simultaneous presentation of two Ps) until a same/different response button was pressed. Correct response times were submitted to a 2 (Memory Task: Spatial vs. Visual) x 2 (Motion Cueing: Motion vs. No Motion) repeated measures ANOVA. There was a significant main effect of Memory Task, $F$ $(1,19)=22.68, M S E=32655.914, p<.001, \eta^{2}=.540$, with response times being longer in the Visual condition $(M=1357 \mathrm{~ms}, S D=311 \mathrm{~ms})$ than in the Spatial condition $(M=1166 \mathrm{~ms}, S D=$ $299 \mathrm{~ms})$. There was no effect of Motion Cueing on Memory Task response time nor was there an interaction between Memory Task and Motion Cueing $(F \mathbf{s}<1$; see Figure 8$)$.

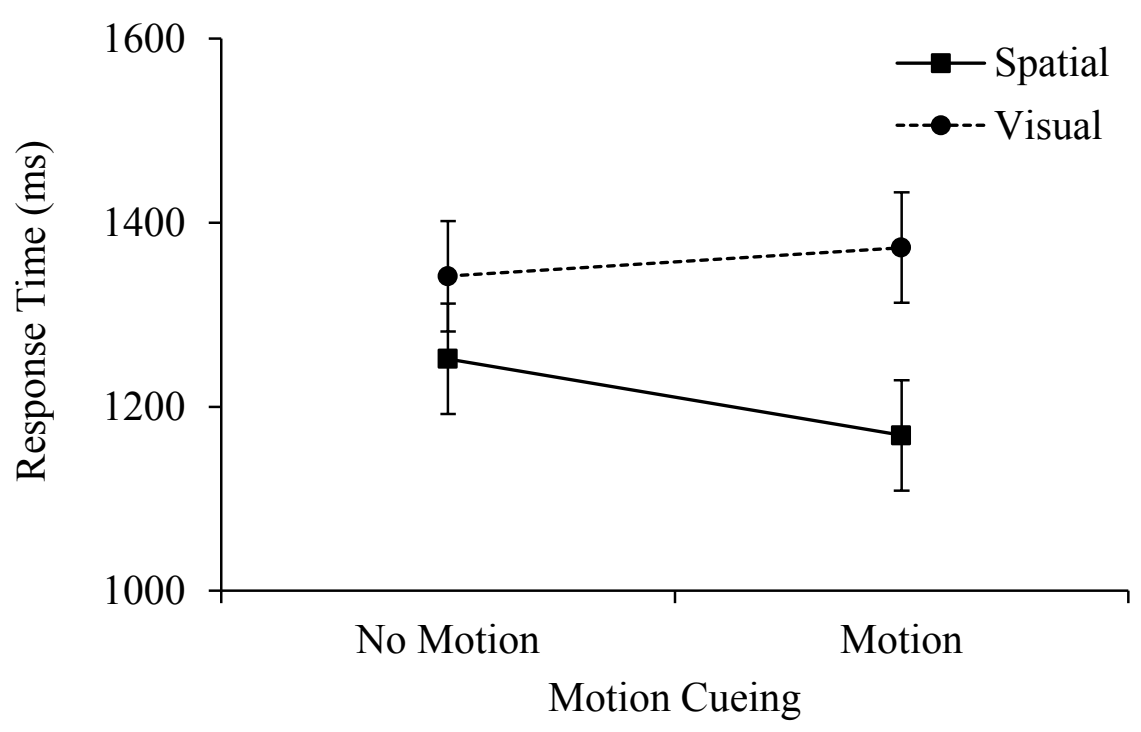

Figure 8. Mean response time (in ms) on the memory task as a function of Motion Cueing and Memory Task in Experiment 1c. Error bars are 95\% confidence intervals. 


\section{Memory Task Accuracy}

Accuracy was recorded as a binary (correct vs. incorrect) response on each memory trial. The accuracy data were submitted to the same ANOVA used to analyze the memory task response time data. The main effect of Memory Task was not significant $(F<1)$. There was a significant main effect of Motion Cueing, $F(1,19)=18.92, M S E=.006, p<.001, \eta^{2}=.499$, with responses being less accurate on trials with motion $(M=67.7 \%, S D=13.0 \%)$ than without $(M=75.3 \%, S D=11.9 \%)$. There was also a significant Memory Task x Motion Cueing interaction, $F(1,19)=4.59, M S E=.004, p=.045, \eta^{2}=.194$. Confidence intervals in Figure 9 indicated that participants were significantly more accurate at remembering location information on trials without motion $(M=75.8 \%, S D=12.0 \%)$ than with motion $(M=68.3 \%, S D=14.6 \%)$. None of the other post-hoc comparisons were significant,

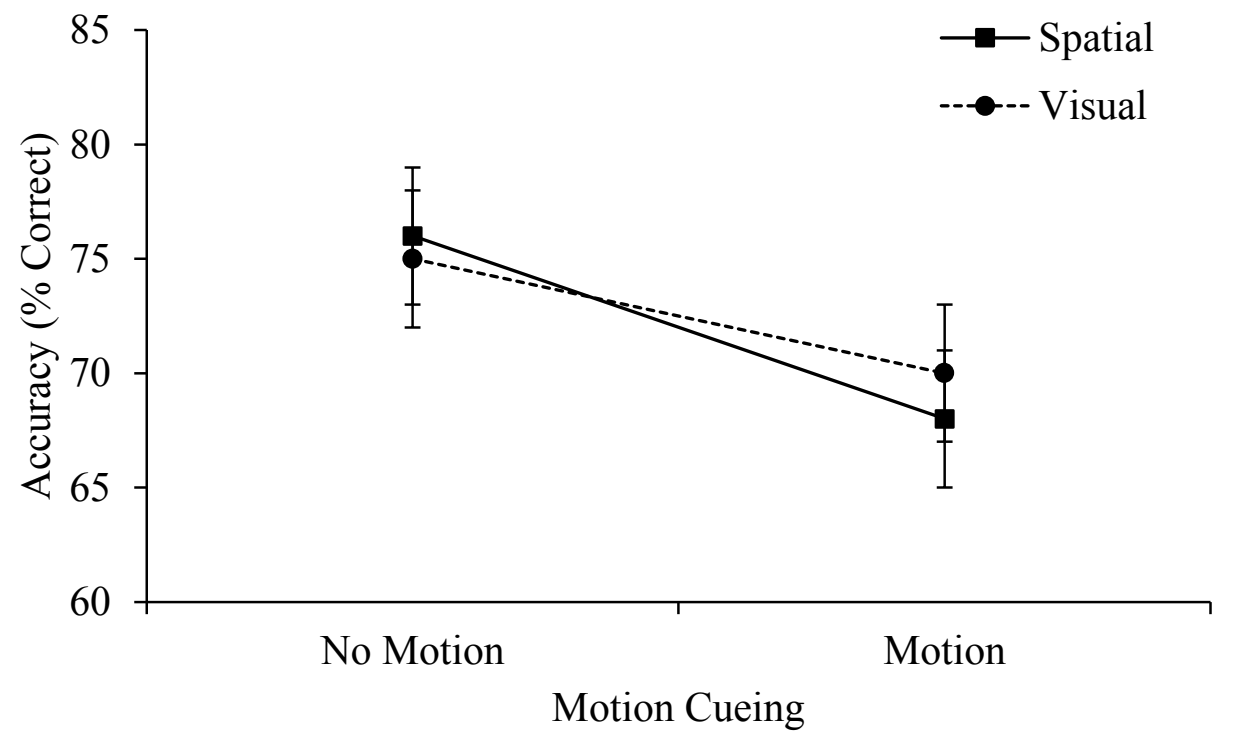

Figure 9. Mean accuracy (\% correct) on the memory task as a function of Motion Cueing and Memory Task in Experiment 1c. Error bars are 95\% confidence intervals. 


\section{Summary}

In summary, Experiment 1c demonstrated the hypothesized greater interference on the spatial memory task when participants discriminated between motion cues. This interference was not bi-directional in that there was no interference from the spatial memory task on the motion cueing task.

\section{Discussion}

Experiment 1 supports the hypothesis that responding to directional motion cues selectively interferes with retention of a concurrent spatial WM task. In particular, in Experiment 1c accuracy on the spatial memory tasks was lower when participants were required to discriminate motion cues $(68 \%)$ than when no motion cues were presented $(76 \%)$. The motion discrimination task did not affect performance on the visual memory task. This pattern of results is consistent with the view that motion processing hinders peoples' ability to rehearse spatial (location) information but not visual (appearance) information. Arguably, this effect occurs because the processing of motion cues and the retention of spatial information compete for the same limited resources in the spatial component of the VSSP.

Although Experiment $1 \mathrm{~b}$ did not yield the expected level of interference of motion on the memory tasks, it demonstrated the feasibility of incorporating the Letter P task in this motioncuing paradigm. The lack of interference from the motion cue discrimination task on the visual and spatial memory task in Experiment $1 \mathrm{~b}$ was likely caused by the motion cueing task being restricted to three cues per trial. Near-ceiling accuracy scores on the motion cue task supported this explanation. In Experiment 1c the number of motion cues was increased to 10 per trial, which required almost continuous responses throughout the $15 \mathrm{~s}$ retention interval. Although the motion cue task was now more difficult, accuracy scores on this task remained high (92\%), 
suggesting that participants were not overwhelmed by the revised version of this task. A posthoc power analysis, using G*Power (Faul, Erdfelder, Buchner, \& Lang, 2009), revealed that in order to have an $80 \%$ chance of finding a significant $(p<.05)$ effect of the memory tasks on motion cue task response times with the current effect size of $\eta^{2}=.108$, a sample of 834 participants would be required.

In sum, Experiment 1 provides evidence that differentiating between motion cues differentially affects visual and spatial memory when the motion occurs during the retention/rehearsal of visual-spatial information. The findings reported in Experiment 1 are, however, limited to the retention of visual/spatial information and therefore do not speak to whether this selective interference effect also occurs during the encoding of visual/spatial information.

\section{Experiment 2}

The purpose of Experiment 2 was to determine whether a motion cue discrimination task selectively interferes with spatial WM during the encoding of visual-spatial information. The encoding durations in Experiment 1 were too short (the target $\mathrm{P}$ display was only shown for 500 ms) to allow a sufficient number of motion cues. As such, the encoding time for the visualspatial displays had to be increased to support the administration of multiple motion cues. This

new requirement resulted in the letter $\mathrm{P}$ task no longer being appropriate because there are only a few ways that Ps can differ (e.g., height, width, thickness). Simply increasing the number of Ps displayed during the extended encoding phase would make the letter P task too difficult given the visual overlap/similarity between the multiple Ps. A new set of visual stimuli was developed to replace the Ps in the letter P task. The new stimuli consisted of multiple nonsense symbols. Given that new stimuli were being used, it was important to establish that the visual and spatial 
WM tasks would be equally difficult. To this end, the difficulty of the visual and spatial memory tasks were equated in Experiment 2a. The effects of motion cueing on these two (equated) memory tasks were then tested in Experiment $2 \mathrm{~b}$.

\section{Experiment 2a}

The purpose of Experiment 2a was to equate the difficulty of the spatial WM task and visual WM task developed for Experiment 2. Performance on these two WM tasks was measured without any motion cueing.

\section{Method}

\section{Participants}

Eight graduate-level colleagues participated without remuneration. Ages ranged from 23 to 32 years $(M=25.4, S D=3.0)$. All participants were right handed and all were assumed to have normal or corrected-to-normal visual acuity.

\section{Design}

The same experimental design used in Experiment la was used here.

\section{Stimulus Materials and Presentation}

The letter P task used in Experiment 1 was replaced with a nonsense symbol task in which several visual symbols without any conventional verbal labels were simultaneously displayed. Like the letter P task, the nonsense symbol task provides a method for manipulating the WM task based on instructions and not by changing the visual stimuli. This ensured that any differences between the spatial and visual WM tasks observed in this experiment cannot be attributed to differences in stimuli and are therefore likely caused by interference from the motion cue discrimination task. Ninety nonsense symbols were randomly selected from a symbol bank with the constraints that there were no highly similar pairs of symbols and that no symbol 
with an obvious verbal label (e.g., @, \#) could be included. The nonsense symbols subtended a visual angle of approximately $6^{\circ}$ both horizontally and vertically.

Location information was manipulated by simultaneously presenting $2,4,6,8$, or 10 target symbols (for $10 \mathrm{~s}$ ) in black ink on a white background each in different randomly selected cells of an invisible 4 x 6 grid. The 4 x 6 grid was not displayed, thus the symbols seemed to appear in random locations on the screen. Following a 3-second retention interval during which visual static filled the screen, the same number of probe symbols were presented simultaneously. On "same" trials, the probe symbols appeared in the same cells as the target symbols. On "different" trials, one of the probe symbols moved to an adjacent cell. The number of candidate cells that a probe symbol could move to varied from one to eight, depending on whether its corresponding target symbol was centered in the grid, along an edge, or in a corner. Only symbols that had at least one empty adjacent cell could change location. Visual (appearance) information was manipulated by changing the identity of one of the symbols. On "same" trials, the probe symbols were the same as the target symbols. On "different" trials, one of the probe symbols changed identity to one of the 90 symbols that was not currently displayed.

\section{Apparatus}

The same apparatus used in Experiment 1a was used here.

\section{Procedure}

Participants were tested in a quiet, distraction-free room. Participants were seated in the motion seat, which was adjusted for height to ensure that their feet rested comfortably on a platform. The seat was then moved forward/backwards and the monitor was moved up/down to hold viewing distance and angle consistent across participants. 
Participants completed a practice session that consisted of 4 Spatial trials and 4 Visual trials, with two same/different trials in each condition. On the Visual trials, a symbol's identity was changed. On the Spatial trials, a symbol's location was changed. Two experimental blocks followed the practice session. One block consisted of 30 Spatial trials and the other consisted of 30 Visual trials with an equal number of same/different trials in each block and block order counterbalanced across participants. The number of symbols displayed started at 2 and increased by 2 after every $5^{\text {th }}$ trial until a maximum of 10 symbols were displayed. Each block lasted approximately 11 minutes with a five-minute break between blocks.

Prior to each experimental block, participants were instructed to remember either the locations (Spatial condition) or the identities (Visual condition) of all the simultaneously presented target symbols. Each trial began with the presentation of a fixation cross for $1 \mathrm{~s}$, followed by the target symbols, chosen at random without replacement from the bank of 90 symbols and displayed in any of the 24 possible screen locations for 10 seconds. Static visual noise that filled the entire monitor was then presented for $3 \mathrm{~s}$. This was followed by a $5 \mathrm{~s}$ presentation of the probe symbols. Participants were required to indicate via button press whether the target and probe symbols were the same or different. 


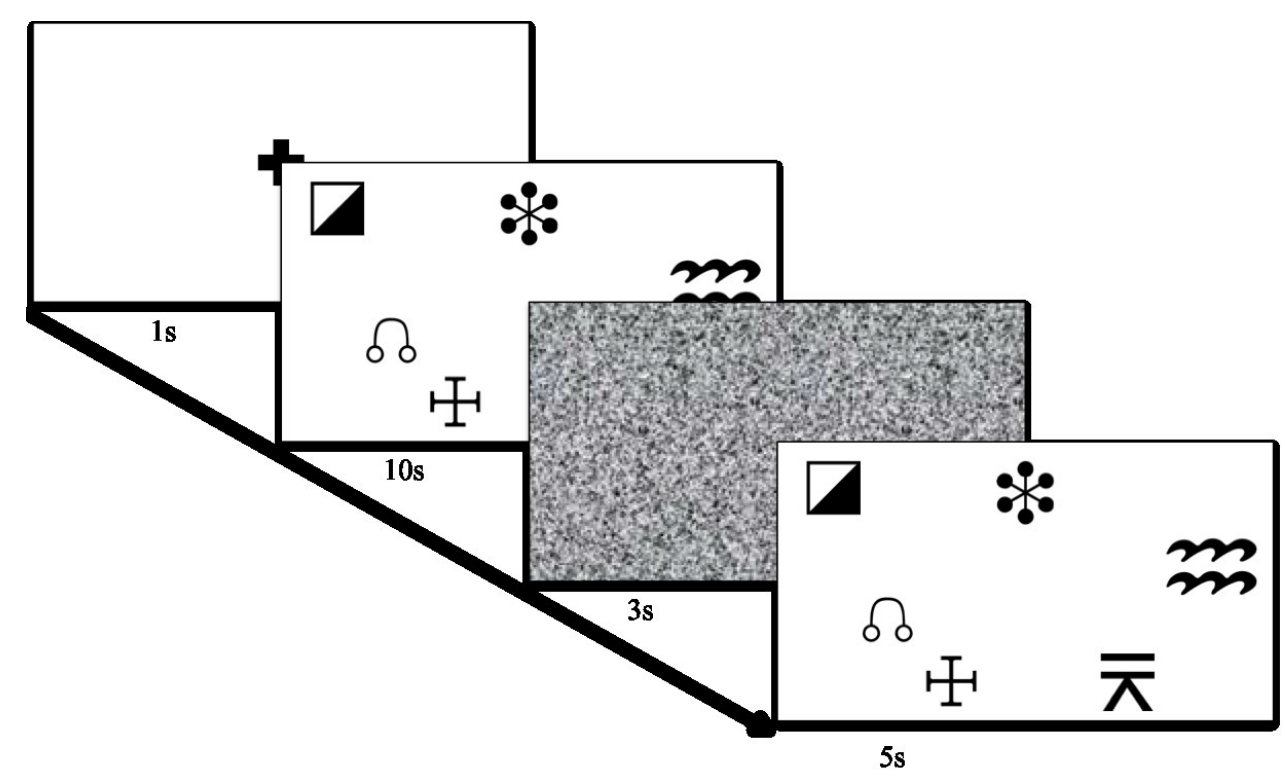

Figure 10. Example of a "same" spatial or visual trial sequence from Experiments $2 \mathrm{a}$ and $2 \mathrm{~b}$.

\section{Results and Discussion}

The purpose of Experiment 2a was to equate Visual and Spatial task difficulty by manipulating the number of symbols displayed and to ensure that performance was above chance but below ceiling. Responses were recorded as either correct or incorrect and accuracy was calculated as the number of correct responses divided by the total number of trials.

The average accuracy rates are presented in Figure 11. As expected, accuracy decreased as the number of symbols increased. Based on these data it was concluded that an accuracy rate of roughly $85 \%$ in both the Spatial and Visual conditions could be achieved by displaying nine and six symbols, respectively. 


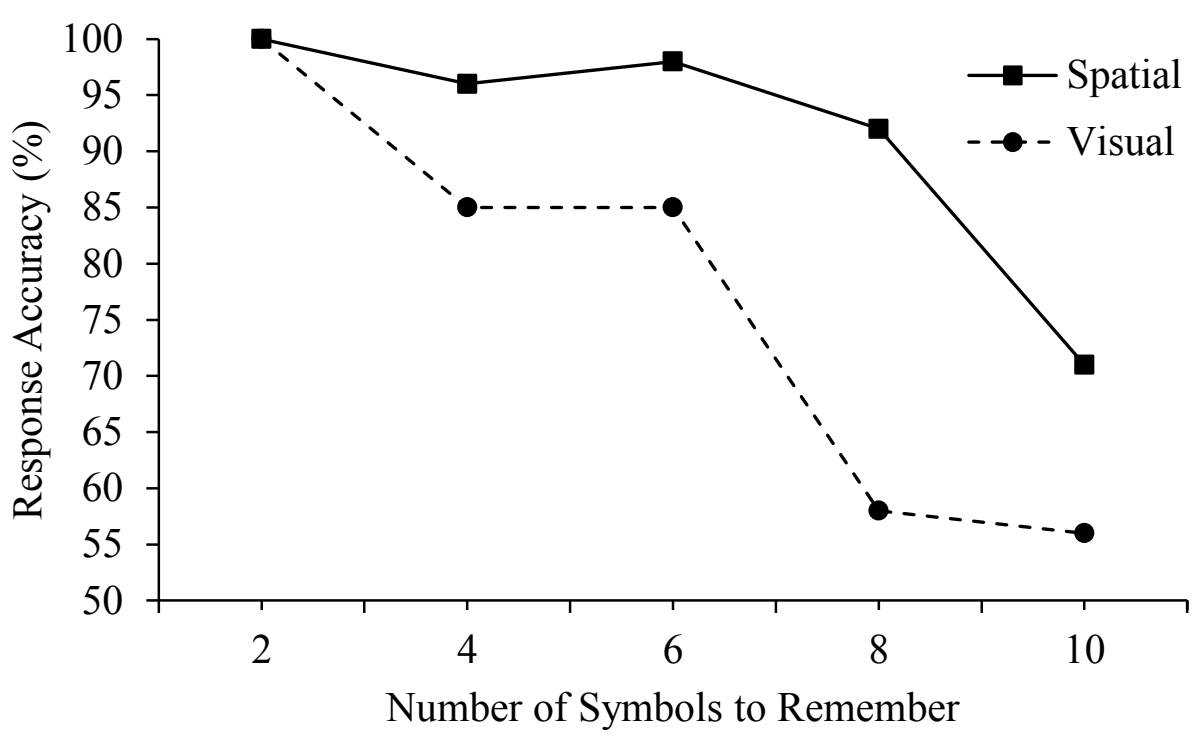

Figure 11. Mean response accuracy (\% Correct) on the memory tasks as a function of Memory Task in Experiment 2a.

\section{Experiment 2b}

The purpose of Experiment $2 \mathrm{~b}$ was to determine whether discrimination between motion cues interferes with the encoding of spatial (location) information and/or visual (appearance) information. It was hypothesized that responding to motion cues would selectively interfere with the Spatial condition. The same visual and spatial WM tasks from Experiment 2a were used. Participants were required to discriminate between 6 directional (left and right) motion cues during the $10 \mathrm{~s}$ encoding interval of the memory trial.

\section{Method}

\section{Participants}

Twenty-four undergraduate students were recruited using Carleton University’s online sign-up system and participated in exchange for $1.0 \%$ course credit. Ages ranged from 17 to 28 years $(M=20.1, S D=2.7)$. Twenty-three participants were right handed and one participant was 
left-handed. No participants reported any history of back injury, concussion, or head injuries and all were assumed to have normal or corrected-to-normal visual acuity.

\section{Design}

The same experimental design used in Experiments $1 \mathrm{~b}$ and $1 \mathrm{c}$ was used here.

\section{Stimulus Materials and Presentation}

The same nonsense symbol task used in Experiment 1a was used here, however, the number of symbols to be remembered on each trial was set at 9 in the Spatial condition and 6 in the Visual condition.

\section{Apparatus}

The same apparatus used in Experiment $1 \mathrm{~b}$ and $1 \mathrm{c}$ was used here.

\section{Procedure}

The experimental set-up was identical to Experiment 2a. Once seated in the motion seat, participants completed a practice session, a motion control condition, and the four experimental conditions created by crossing Motion Cueing (Motion vs. No Motion) and Memory Task (Spatial vs. Visual). The practice session was identical to the practice session in Experiment 2a. The motion control condition required participants to respond left/right to 30 motion cues as fast as they could without a memory task. Motion cues were presented every 1.5 to $5 \mathrm{~s}$ (randomly determined). Participants then completed the four experimental conditions, each of which consisted of 30 trials. The four conditions were blocked and counterbalanced across participants such that each possible condition order was tested. Each block lasted approximately 11 minutes with five-minute breaks between blocks.

Prior to each experimental block, participants were instructed to remember either the location or the visual appearance (font type) of the symbols that would be presented. Six motion 
cues were randomly presented during the 10 -second encoding phase (target display) with the constraints that the minimum inter-cue interval was $500 \mathrm{~ms}$ and the maximum inter-cue interval was $7000 \mathrm{~ms}$ (the average inter-cue interval was $1800 \mathrm{~ms}$ ). There was also a minimum of $500 \mathrm{~ms}$ between the onset of the target display and the first cue, and between the last cue and the $3 \mathrm{~s}$ retention phase. Participants were instructed to respond as quickly as possible to the six motion cues by pressing the appropriate button(s) on the response pad.

\section{Results and Discussion}

The same four measures of interest in Experiment $1 b$ and $1 c$ are reported here in the following subsections.

\section{Motion Cue Response Time}

Response time was measured as the difference (in ms) between the initiation of the seat's motion and the participant's button press response. Each participant responded to 390 motion cues in total. Thirty motion cues were presented in isolation in the motion control condition to establish baseline performance. The remaining 360 motion cues occurred in the 30 spatial and 30 visual trials in the "Motion" condition, with six motion cues being presented on each trial. Only correct responses to the motion cues were included in the response time analysis. The data were submitted to a one-factor (Memory Task: Baseline (no WM task) vs. Spatial vs. Visual) repeated measures analysis of variance (ANOVA). The effect of Memory Task was not significant, $F$ $(2,46)=2.59, M S E=1500.613, p=.086, \eta^{2}=.101$.

\section{Motion Cue Accuracy}

Accuracy was recorded as a binary (correct vs. incorrect) response to each motion cue. These data were submitted to the same repeated measures ANOVA used in the motion cue 
response time analysis. The effect of Memory task was not significant $(F<1)$, with no statistical differences between the Baseline $(M=85.0 \%, S D=24.0 \%)$, Spatial $(M=87.2 \%, S D=$ $25.8 \%)$, and Visual $(M=86.9 \%, S D=26.6 \%)$ conditions.

\section{Memory Task Response Time}

Response times were measured (in ms) from the onset of the probe display until a same/different response button was pressed. Correct response times were submitted to a 2 (Memory Task: Spatial vs. Visual) x 2 (Motion Cueing: Motion vs. No Motion) repeated measures ANOVA. There was a significant main effect of Memory Task, $F(1,23)=36.37, M S E$ $=87392.015, p<.001, \eta^{2}=.614$, with response times being longer on the Visual task $(M=$ $1973 \mathrm{~ms}, S D=897 \mathrm{~ms})$ than on the Spatial task $(M=1606 \mathrm{~ms}, S D=848 \mathrm{~ms})$. The main effect of Motion Cueing was not significant $F(1,23)=1.35, M S E=65539.152, p=.258, \eta^{2}=.055$. There was no interaction between Memory Task and Motion Cueing $(F<1$; see Figure 12).

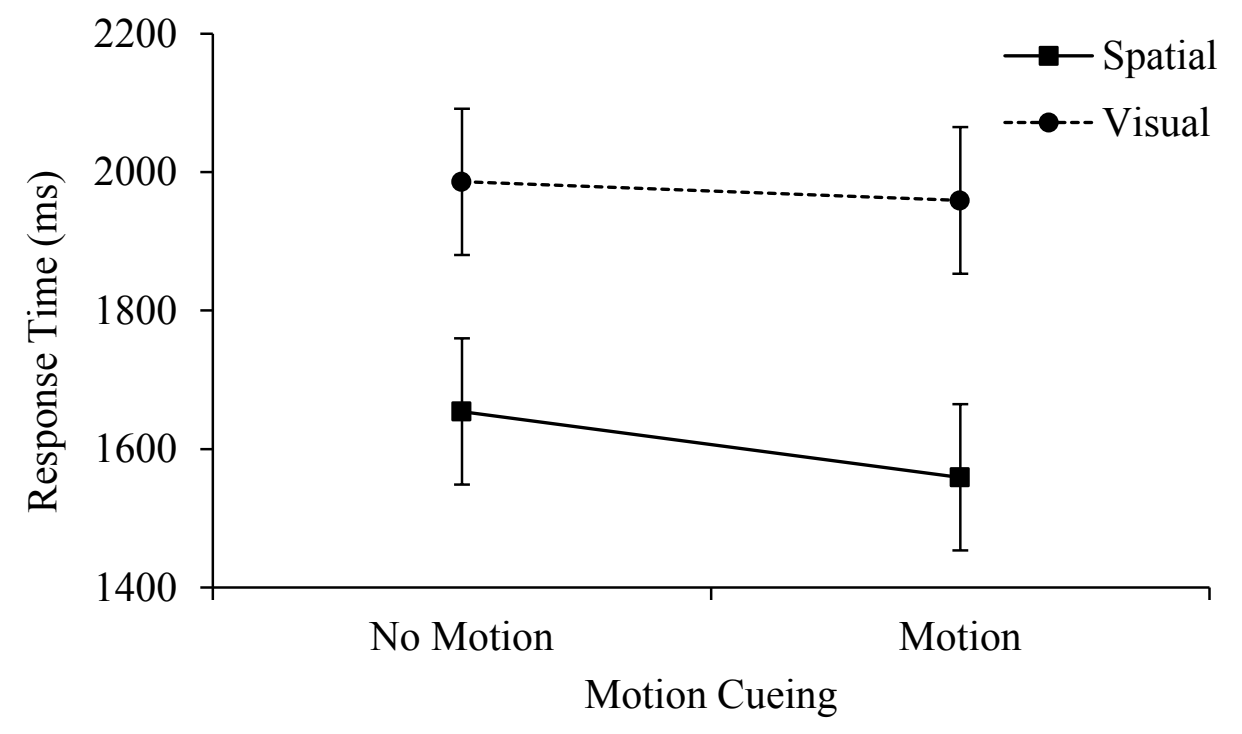

Figure 12. Mean response time (in ms) on the memory task as a function of Motion Cueing and Memory Task in Experiment 2b. Error bars are 95\% confidence intervals. 


\section{Memory Task Accuracy}

Accuracy was recorded as a binary (correct vs. incorrect) response on each memory trial. The accuracy data were submitted to the same ANOVA used to analyze the memory task response time data. The main effect of Memory Task was significant $F(1,23)=9.48, M S E=$ $.008, p=.005, \eta^{2}=.292$. Memory accuracy scores were significantly higher in the Spatial condition $(M=80.3 \%, S D=14.9 \%)$ than in the Visual Condition $(M=74.8 \%, S D=11.6 \%)$. There was a significant main effect of Motion Cueing, $F(1,23)=24.01, M S E=.006, p<.001$, $\eta^{2}=.551$. Memory trial accuracy scores were significantly higher in the No-Motion condition $(M$ $=81.5 \%, S D=12.2 \%)$ than in the Motion condition $(M=73.5 \%, S D=12.7 \%)$. As can be seen in Figure 13, there was a significant Memory Task x Motion Cueing interaction, $F(1,19)=$ $11.73, M S E=.004, p=.002, \eta^{2}=.338$. Confidence intervals in Figure 13 indicate that participants were significantly more accurate at remembering spatial information on trials without motion $(M=86.4 \%, S D=10.5 \%)$ than with motion $(M=74.2 \%, S D=14.4 \%)$. Also, participants were more accurate at remembering spatial information $(M=86.4 \%, S D=10.5 \%)$ than visual information $(M=76.7 \%, S D=12.0 \%)$ when no motion cues were present. No other mean accuracy scores were statistically different. 


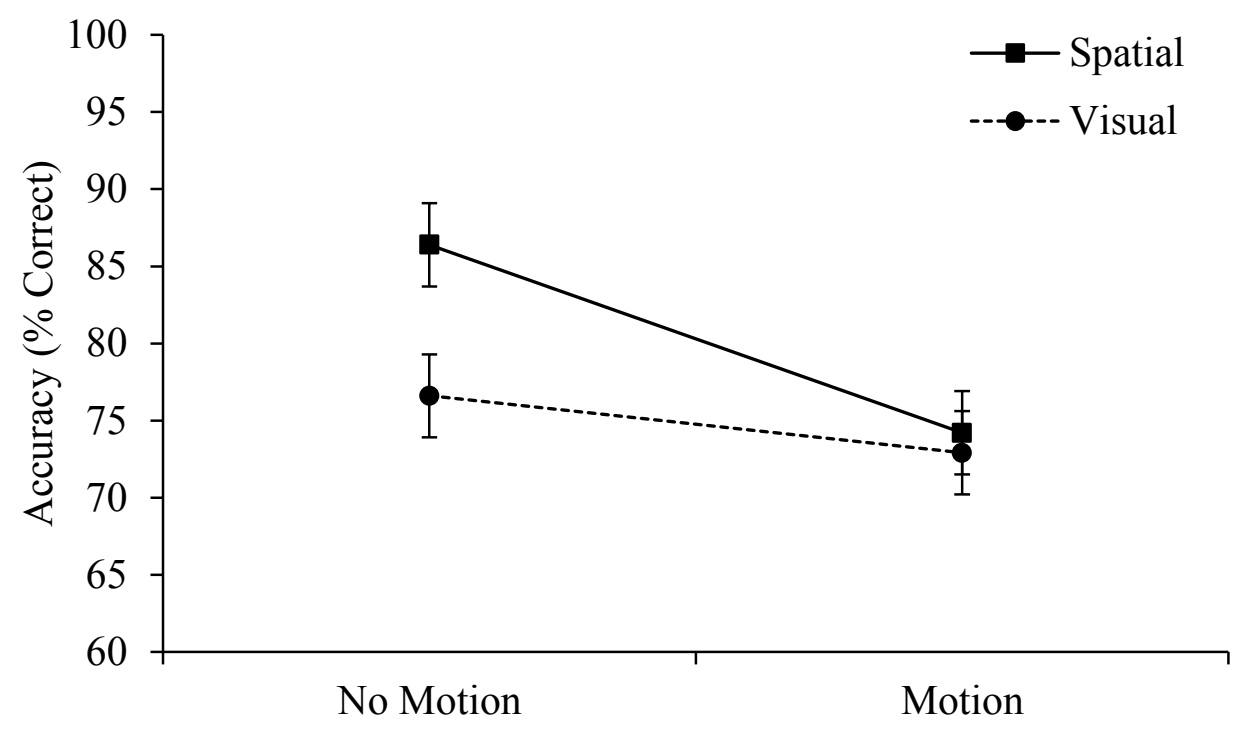

Figure 13. Mean accuracy (\% Correct) on the memory task as a function of Motion Cueing and Memory Task in Experiment 2b. Error bars are 95\% confidence intervals.

\section{Summary}

In summary, Experiment $2 \mathrm{~b}$ demonstrated the hypothesized selective interference to the Spatial memory condition when participants discriminated between motion cues. No interference on the motion cueing task (accuracy or response latency) was observed in either of the memory tasks conditions.

\section{Discussion}

The purpose of Experiment 2 was to extend the findings of Experiment 1 by exploring whether or not the motion cueing task selectively interfered with spatial WM when the motion cues were presented during the encoding of the spatial information rather than during retention. The significant Motion Cueing x Memory Task interaction observed in Experiment 2b supports the hypothesis that processing motion cues interferes with the concurrent encoding of spatial information but not visual information. That is, accuracy on the spatial memory task was 
significantly lower when paired with motion while accuracy on the visual memory task was unaffected by motion.

In order to present a sufficient number of motion cues, the encoding phase of the memory tasks was increased from $750 \mathrm{~ms}$ in Experiment 1 to $10 \mathrm{~s}$ in Experiment 2. The difficulty of the memory tasks had to be increased to be commensurate with the increased encoding time. Rather than displaying more Ps, which have limited variability in terms of their visual appearance, the letter P task was replaced with a nonsense symbol task. The nonsense symbol task allowed more stimuli to be presented during the extended encoding phase, but because the variability in visual appearance between the nonsense symbols was greater than that for the Ps, participants were still capable of making fast and accurate responses to the spatial and visual memory tasks.

Given that the $10 \mathrm{~s}$ encoding time in Experiment 2 was shorter than the $15 \mathrm{~s}$ retention interval in Experiment 1, only 6 motion cues (instead of 10) per trial were administered in Experiment 2. Overall, accuracy on the motion cue discrimination task in Experiment 2 (96\%) was similar to that reported in Experiment 1. As in Experiment 1, there was no effect of the memory tasks on the response times to the motion cue discrimination task in Experiment 2. A post hoc power analysis, using $\mathrm{G}^{*}$ Power (Faul et al., 2009), revealed that in order to have an $80 \%$ chance of finding a significant $(\mathrm{p}<.05)$ effect of the memory tasks on motion cue task response times with the current effect size of $\eta^{2}=.101$, a sample of 951 participants would be required.

As in Experiment 1, there was an overall significant main effect of motion cueing on the memory task accuracy rates with higher accuracy in the No Motion condition $(86 \%)$ than in the Motion condition (74\%). This finding shows that processing motion cues interferes with the encoding of information into WM. There was also a significant main effect of Memory Task in 
both the response time and accuracy data, with higher performance in the Spatial condition than in the Visual condition. This main effect was not expected given that efforts were made to equate the difficulty of these two tasks in Experiment 2a. That said, although this main effect of Memory Task is not ideal, it does not pose a major problem as the primary goal of this thesis is to determine the effects of motion cueing within each of the two types of memory tasks.

In sum, Experiment $2 \mathrm{~b}$ provides clear evidence that encoding spatial information (visually) and discriminating between directional motion cues compete for WM resources as demonstrated by decrease in memory task accuracy. As predicted, no significant interference effects were observed in the visual memory task by discrimination of motion cues.

\section{General Discussion}

The present research examined the link between visual-spatial WM and motion cueing. Two experiments were conducted in which a dual-task paradigm was used to assess the extent to which a motion cue discrimination task interfered with the rehearsal (E1) and the encoding (E2) of visual and spatial information. It was hypothesized that because participants were instructed to place equal effort on completing both the motion cueing task and the memory task, interference would be bi-directional. That is, processing motion cues was expected to impair the rehearsal/encoding of spatial information and the rehearsal/encoding of spatial information was expected to impair the processing of motion cues. Experiments 1 and 2 revealed that the motion cue task impaired memory (accuracy) for the retention and encoding of spatial information, but not visual information.

This thesis is one of the few attempts to use a WM framework to constrain and understand the applied issue of using motion in simulator training. To date, research has tended to be focused on high-level measures such as the control and handling of the aircraft (de Winter, 
Dodou, \& Mulder, 2012) while neglecting the cognitive limitation of the operator. A better understanding of the cognitive limitations of operators will lead to more directed research in the field of flight simulation development. Results from the current studies yield important information about WM as well as the impacts of simulator motion on WM.

\section{Working Memory}

In Baddeley's model of WM (Baddeley, 2000; 2012), spatial location and motion information is processed by the inner scribe whereas visual information (e.g., form, appearance) is stored by the visual cache. The data reported here support Baddeley's conceptualization of separable modules for the storage of location-based information versus appearance-based information. The current findings support the fractionation of the VSSP into visual and spatial subcomponents. That is, the discrimination of motion cues selectively interfered with the encoding and retention of location-based information but not appearance-based information.

Results from the current research add to the base of behavioural, developmental, imaging and special population studies that suggest visual memory and spatial memory are distinctly different subcomponents of visual-spatial WM. Experiment 1 was a quasi-replication of the Darling et al. (2007) study where spatial tapping interfered with spatial WM but not visual WM. However, one limitation of the Darling et al. study was that the accuracy of the interference task (spatial tapping) could not be measured. This limitation was overcome in the current research by measuring both the response accuracy and response latency to the motion cues. Measuring accuracy on both tasks (motion cueing and memory task) allowed detection of bi-directional interference (E1b) in the motion/spatial condition only. Responding to motion cues reduced the accuracy of the spatial memory task whereas accuracy on the motion cuing task was also negatively affected by the spatial memory task. 
Thomas and Seiffert (2010) demonstrated that active and passive self-motion interferes with the ability to track objects (spatial task), however, these effects occurred only after the tracking task has reached some level of difficulty. When Thomas and Seiffert's participants only tracked one object there was no negative impact of self-motion but when they increased the objects to three, interference began to be observed. A similar threshold was discovered in the current research. In Experiment 1b participants where required to respond to three motion cues and this yielded minimal interference on the spatial memory task (accuracy of $78 \%$ ). In Experiment $1 \mathrm{c}$ the number of motion cues was increased to ten and the accuracy on the spatial memory task decreased by $10 \%$. Taken together, both the current research and Thomas and Seiffert's research demonstrate that having two tasks that merely utilize the same resource is not enough to cause measurable interference. It would seem that there is a capacity to the spatial WM component and that capacity must be reached or exceeded before significant dual-task interference is observed.

The current findings also relate to the interaction between aspects of WM and postural control (Lajoie et al., 1993; Makizako et al., 2013; Maylor \& Wing, 1996). Postural control can be negatively affected by tasks that require spatial WM (Makizako et al., 2013; Woollacott \& VanderVelde, 2008), and attempts to maintain postural control can negatively affect performance on spatial WM tasks (VanderVelde, Woollacott, \& Shumway-Cook, 2005). There is the possibility that the current findings were at least partly due to the interference motion cues can cause on postural control. Although participants were seated (with no way of falling), the stability of their orientation was constantly being disrupted. If, as in postural control, there was an automatic 'posture first' response it would likely draw spatial resources away from the spatial WM task. 
Motion simulator research also supports the notion that producing instability in ones' sense of orientation or motion can selectively interfere with spatial WM. When Gresty et al. (2003) had participants perform a spatial and verbal WM task in a motion simulator that provided stable and congruent visual and motion movements there was little negative impact on either WM task. However, when the physical motion was opposite of the visual motion (instability of motion perception between the two modalities) performance on the spatial, but not the verbal task, suffered. Gresty et al.'s results were obtained with no requirement of the participants to actively monitor the motion of the simulator. Therefore, it is not surprising that the findings of the current research, which required participants to actively respond to both tasks, are consistent with the idea that the presence of simulator motion does require spatial WM capacity.

\section{Flight Simulation}

The finding that discriminating between motion cues interferes with the encoding and rehearsal of spatial information in WM has direct applicability to the debate surrounding the need for motion in flight simulators. Basing requirements for motion in simulation-based training solely on subjective claims regarding its positive impact on simulation fidelity is tenuous. When considering the cost and complexity of motion flight simulators, as well as the safety risks associated with the potential interfere with the training of operators (specifically, the possibility of introducing information that is uninformative, or worse, confusing), a more rigorous investigation must take place. Objective data should be used to support these subjective claims when deciding whether or not to include motion in simulation. Unfortunately, because high-level quantitative data is often hard to obtain and subsequently to interpret, more attempts to understand how fundamental cognitive processes (including attention and memory) are involved 
in the perception and processing of motion information should be made. The current research highlights two arguments, one in favour of the inclusion of motion in simulators and one partially against it.

Experiments 1 and 2 both demonstrated that discriminating between directional motion cues requires cognitive resources that appear to selectively overlap (interfere) with spatial WM. When flying under manual control in a real flight environment, pilots are constantly correcting attitude, sometimes according to their instruments and sometimes according to feedback from motion. In cases where pilots are using motion-induced forces exerted on the body to monitor the aircraft's orientation, it follows from the results of the current research that any spatially-based task (e.g., map reading, path planning, remembering the form and location of symbols on a display), will be impaired relative to when these motion cues are not present. Furthermore, any visually-based tasks (e.g., remembering the appearance of visual references, numerical data, or gauge readings) will be relatively unaffected by motion information. If pilots are training in simulators without motion, then the cognitive resources used to monitor and respond to motion may be free to support spatial tasks resulting in an underestimation of the workload required to complete the same task during real flight. This argument therefore supports the idea that motion should be included in simulators, if not to increase immersion, transfer of training, or aircraft handling, then at the very least to accurately represent the cognitive demands associated with processing motion in real flight.

Based on this argument for the inclusion of motion in simulators, the experience level of the pilot in training must also be considered. Previous research has illustrated a difference in the benefits of motion (on specific flight tasks) across experience level (Noble, 2002). On tasks requiring pilots to react to disturbance motion (turbulence or unstable flight), simulator motion 
was more beneficial for novice pilots than for experienced pilots. Given that novice pilots are not entirely familiar with how their control inputs will affect the attitude of the aircraft (de Winter et al., 2012), motion feedback provides additional information that may improve their performance. However, based on the previous logic, if the monitoring of motion information requires extra cognitive capacity it could be overwhelming to very inexperienced pilots. Therefore, when training novice pilots on tasks not related to disturbance motion, it may be beneficial to omit motion until they have reached some level of competency on basic aircraft handling.

\section{Strengths and Limitations}

There were two main limitations to the current experiments. First, the design of the dynamic motion seat restricted the amount of motion displacement. The seat is designed to run a full flight model, thus it is difficult, but not impossible, to isolate motion channels. Here, the command to bank left or right using the seat pan and seat back provided the discrete left/right motion cues. Although the displacement of the seat pan and back (approximately $2.5 \mathrm{~cm}$ off level in each direction) was known and consistent across conditions and experiments, the magnitude (velocity) of the cues was unknown because the exact duration over which the cue was applied was unavailable. However, it was known that the motion cue was executed in less than one second. This limitation was likely not important for the current investigation as the main purpose of the motion cueing was to provide consistent proprioceptive direction cues. It may prove valuable to investigate the role of the magnitude of the motion cues in future research. It may be that more salient (greater displacement or longer duration) cues require less spatial WM to monitor, whereas less salient or possibly conflicting cues may require more spatial WM. 
Response latencies were longer in the Visual (font type, stimulus identity) condition than in the Spatial (location) condition in both experiments. This was the case even when there were more stimuli to remember in the Spatial condition (9) than in the Visual condition (6) in Experiment 2. However, participants' responses in the Visual conditions were still sufficiently fast that they did not exceed the time allotted to respond ( $3 \mathrm{~s}$ in E1 and $5 \mathrm{~s}$ E2) and therefore did not influence accuracy rates. The difference in response latencies may have been due to different strategies when checking the probe items. That is, it is possible that on spatial trials, participants encoded the stimuli as a whole and were able to quickly determine if a part of the whole (an individual symbol) moved. However, on visual memory trials, participants likely had to examine each symbol in a serial fashion to determine if its font/identity had changed.

\section{Future Directions}

The primary function of dynamic motion seats is to provide discreet motion cues. The more widely used Stewart platform is capable of providing longer duration and larger displacements cues allowing for a more full-body movement (de Groot et al., 2011). Given that most motion simulators use Stewart platforms to induce motion, an important next step would be to attempt to replicate the current findings in a continuous motion environment rather than the discrete motion cue as was used here. Relative to processing discrete motion cues, monitoring continuous motion is likely to require more (or at least more sustained) effort and would therefore produce more interference on a secondary spatial task. Exploring the cognitive processing differences between discrete motion cues and full body motion experienced on a Stewart platform is important because if it can be demonstrated that discrete directional cues are cognitively the same as full motion, then providing complex and costly motion is unnecessary to simulate the cognitive demands associated with processing motion in real flight. 
Finally, the use of motion cueing in the current studies presents a new paradigm for studying spatial WM. The way in which the dynamic motion seat was utilized for the current research was one of many ways in which it could have been used to load spatial WM. Extending similar findings (Darling et al., 2009) with a completely new method provides evidence for the robustness of the WM frameworks proposed by Baddeley (Baddeley, 1984; 1986; 1988; 1992; 2012; Baddeley \& Hitch, 1974).

The current research helps to fill gaps in the literature between full-mission flight simulation research and basic WM research. The findings demonstrate that basic research can be useful to the simulation community. In a continuing attempt to improve flight simulators and make educated decisions on the inclusion of motion in simulators, the ways in which motion affects the operator must be understood. If motion does not provide additional training benefit there is no need to spend time and money on the inclusion of motion. The exceptions are situations related to disturbance motion in which aircraft handling performance has been shown to benefit from motion (de Winter et al., 2012). However, as demonstrated by the results of the current research, monitoring and responding to directional motion cues does cause interference to WM tasks, specifically spatial tasks, and therefore there is clear motivation to consider including motion in simulator-based training. 


\section{References}

Allen, R. J., Baddeley, A. D., \& Hitch, G. J. (2014). Evidence for two attentional components in visual working memory. Journal of Experimental Psychology: Learning, Memory, and Cognition, doi:http://dx.doi.org/10.1037/xlm0000002

Baddeley, A. (1984). The fractionation of human memory. Psychological Medicine, 14, 259264. doi:10.1017/S0033291700003536

Baddeley, A. (1986). Working Memory. Oxford: Oxford University Press.

Baddeley, A. (1988). Cognitive psychology and human memory. Trends in Neurosciences, 11, $176-181$.

Baddeley, A. (1992). Working memory. Science, 225, 556-559.

Baddeley, A. (1996). The fractionation of working memory. Proceedings of the National Academia of Science, 93, 13468-13472.

Baddeley, A. (2000). The episodic buffer: A new component of working memory? Trends in Cognitive Sciences, 4, 417-423.

Baddeley, A. (2012). Working memory: theories, models, and controversies. Annual review of psychology, 63, 1-29. doi:10.1146/annurev-psych-120710-100422

Baddeley, A. D., \& Hitch, G. (1974). Working Memory. In G. H. Bower (Ed.), (Vol. 8, pp. 47 89). Academic Press. doi:http://dx.doi.org/10.1016/S0079-7421(08)60452-1

Been-Lirn Duh, H., Parker, D. E., Philips, J. O., \& Furness, T. A. (2004). “Conflicting” motion cues to the visual and vestibular self-motion system around $0.06 \mathrm{~Hz}$ evoke simulator sickness. The Journal of Human Factors and Ergonomics Society, 46, 142-153. doi: 10.1518/hfes.46.1.142.30384 
Berger D. R., \& Bulthoff, H. H. (2009). The role of attention on the integration of visual and inertial cues. Experimental Brain Research, 198, 287-300. doi: 10.1007/s00221-009$1767-8$

Burki-Cohen, J., Soja, N. N., \& Longridge, T. (1998). Simulator platform motion - The need revised. The International Journal of Aviation Psychology, 8, 293 - 317.

Bürki-Cohen, J., Boothe, E. M., Soja, N. N., DiSario, R. D., Go, T., \& Longridge, T. (2000, May). Simulator fidelity: The effect of platform motion. In proceedings of the Royal Aeronautical Society conference on Flight Simulation-The Next Decade, London.

Cocchini, G., Logie, R. H., Sala, S. D., MacPherson, S. E., \& Baddeley, A. (2002). Concurrent performance of two memory tasks: Evidence for domain-specific working memory systems. Memory \& Cognition, 30, 1086-1095.

Darling, S., Sala, D. S., \& Logie, R. H. (2007). Behavioral evidence for separating components within visuo-spatial working memory. Cognitive Processing, 8, 175-181. doi: 10.1007/s10339-007-0171-1

Darling, S., Sala, D. S., \& Logie, R. H. (2009). Dissociation between appearance and location within visuo-spatial working memory. The Quarterly Journal of Experimental Psychology, 62, 417-425. doi: 10.1080/17470210802321984

Darling, S., Della Sala, S., Logie, R. H., \& Cantagallo, A. (2006). Neuropsychological evidence for separating components of visuo-spatial working memory. Journal of Neurology, 253, 176-80. doi:10.1007/s00415-005-0944-3

Della Sala, S., Gray, C., Baddeley, A., Allamano, N., \& Wilson, L. (1999). Pattern span: a tool for unwelding visuo-spatial memory. Neuropsychologia, 37, 1189-99. 
de Groot, S., de Winter, J. C. F., Mulder, M., \& Wieringa, P. A. (2011). Nonvestibular motion cueing in a fixed-base driving simulator: Effects on driver breaking and cornering performance. Presence, 20, 117-144.

de Winter, J. C. F., Dodou, D., \& Mulder, M. (2012). Training effectiveness of whole body flight simulator motion: A comprehensive meta-analysis. The International Journal of Aviation Psychology, 22, 164-183. doi: 10.1080/10508414.2012.663247

Engel, A., Burke, M., Fiehler, K., Bien, S., \& Rösler, F. (2008). Motor learning affects visual movement perception. European Journal of Neuroscience, 27, 2294-2302.

Engle, R. W., Kane, M. J., \& Tuholski, S. W. (1999). Individual differences in working memory capacity and what they tell us about controlled attention, general fluid intelligence, and functions of the prefrontal cortex. In A. Miyake \& P. Shah (Eds.), Models of working memory (pp.102-134). New York: Cambridge University Press.

Ernst, M. O., \& Bulthoff, H. H. (2004). Merging the senses into a robust percept. Trends in Cognitive Science, 8, 162-169.

Farmer, E. W., Berman, J. V. F., \& Fletcher, L. (1986). The Quarterly Journal of Experimental Psychology Section A : Human Experimental Psychology Evidence for a visuo-spatial scratch-pad in working memory. The Quarterly Journal of Experimental Psychology, 38, $675-688$.

Faul, F., Erdfelder, E., Buchner, A., \& Lang, A.G. (2009). Statistical power analyses using G*Power 3.1: Tests for correlation and regression analyses. Behavior Research Methods, $41,1149-1160$ 
Fiechler, K., Burke, M., Engel, A., Bien, S., \& Rosler, F. (2008). Kinistetic working memory and action control within the dorsal stream. Cerebral Cortex, 18, 243-253. doi: 10.1093/cercor/bhm071

Fougnie, D., \& Marois, R. (2006). Distinct capacity limits for attention and working memory. Psychological Science, 17, 526-534.

Goble, D. J., Aaron, M. B., Warschausky, S., Kaufman, J. N., \& Hurvitz, E. A. (2012). The influence of spatial working memory on ipsilateral remembered proprioceptive matching in adults with cerebral palsy. Experimental Brain Research, 223, 259-69. doi:10.1007/s00221-012-3256-8

Gresty, M. A., \& Golding, J. F. (2009). Impact of vertigo and spatial disorientation on concurrent cognitive tasks. Basic and Clinical Aspects of Vertigo and Dizziness: Annals of the New York Academy of Sciences, 1164, 263-267.

Gresty, M. A., Waters, S., Bray, A., Bunday, K., \& Golding, J. F. (2003). Impairment of spatial cognitive function with preservation of verbal performance during spatial disorientation. Current Biology, 13, 829-831. doi: 10.1016/j.cub.2003.10.013

Hale, S., Myerson, J., Rhee, S. H., Weiss, C. S., \& Abrams, R. A. (1996). Selective interference with the maintenance of location information in working memory. Neuropsychology, 10, 228-240. doi:10.1037//0894-4105.10.2.228

Hanes, D. A., \& McCollum, G. (2006). Cognitive-vestibular interactions: A review of patient difficulties and possible mechanisms. Journal of Vestibular Research, 16, 75-91

Ikkai, A., \& Curtis, C. E. (2011). Common neural mechanisms supporting spatial working memory, attention and motor intention. Neuropsychologia, 49, 1428-34. doi:10.1016/j.neuropsychologia.2010.12.020 
Jarmasz, J., \& Hollands J. G. (2009). Using confidence intervals in repeated-measures designs: The number of observations principle. Canadian Journal of Experimental Psychology, 63, $124-138$.

Lajoie, Y., Teasdale, N., Brad, C., \& Fleury, M. (1993). Attentional demands for static and dynamic equilibrium. Experimental Brain Research, 97, 139-144.

Lawrence, B. M., Myerson, J., \& Abrams, R. A. (2004). Interference with spatial working memory: an eye movement is more than a shift of attention. Psychonomic Bulletin \& Review, 11, 488-94.

Lawrence, B. M., Myerson, J., Oonk, H. M., \& Abrams, R. A. (2001). The effects of eye and limb movements on working memory. Memory, 9, 433-444. doi:10.1080/09658210143000047

Loftus, G. R., \& Masson, M. E. (1994). Using confidence intervals in within-subject designs. Psychonomic Bulletin \& Review, 1, 476-490. doi:10.3758/BF03210951

Logie, R. H. (1995). Visuo-spatial Working Memory. Lawrence Erlbaum. Retrieved from http://books.google.ca/books?id=AoIZA4VqpcoC

Logie, R. H. (2011). The functional organization and capacity limits of working memory. Current Directions in Psychology, 20, 240-245. doi: 10.1177/0963721411415340

Logie, R. H., \& Baddeley, A. D. (1987). Cognitive processes in counting. Journal of Experimental Psychology, 13, 310-326.

Logie, R. H., \& Pearson, D. G. (1997). The Inner Eye and the Inner Scribe of Visuo-spatial Working Memory : Evidence from Developmental Fractionation. European Journal of Cognitive Psychology, 9, 241-257. 
Makizako, H., Furuna, T., Ihira, H. \& Shimada, H. (2013). Age-related differences in the influence of cognitive task performance on postural control under unstable balance conditions. International Journal of Gerontology, 1-6. doi: 10.1016/j.ijge.2013.01.014.

Maylor, E. A., \& Wing, A. M. (1996). Age differences in postural stability are increased by attritional cognitive demands. Journal of Gerontology: Psychological Sciences, 51, 143154.

McCauley, M. E. (2006). Do Army helicopter training simulators need motion bases? (Tech. Rep. No. 1176). Arlington, VA: U.S. Army Research Institute for the Behavioral and Social Sciences.

Noble, C. (2002). The relationship between fidelity and learning in aviation training and assessment. Journal of Air Transportation, 7, 33-54.

Pickering, S. J., Gathercole, S. E., Hall, M., \& Lloyd, S. A. (2001). Development of memory for pattern and path : Further evidence for the fractionation of visuo-spatial memory. The Quarterly Journal of Experimental Psychology, 54, 397-420. doi:10.1080/0272498004200017

Postle, B. R., D’Esposito, M., \& Corkin, S. (2005). Effects of verbal and nonverbal interference on spatial and object visual working memory. Memory and Cognition, 33, 203-212.

Postle, B. R., Idzikowski, C., Della Sala, S., Logie, R. H., \& Baddeley, A. D. (2006). The selective disruption of spatial working memory by eye movements. Quarterly Journal of Experimental Psychology, 59, 100-120. doi:10.1080/17470210500151410.The

Qualification Performance Standards for Airplane Full Flight Simulators, FAA-2002-12461, 73 FR 26490 (2013) 
Ramenzoni, V. C., Riley, M. A., Shockley, K., \& Chiu, C. Y. P. (2007). Postural response to specific types of working memory tasks. Gait \& Posture, 25, 368-373. 10.1016/j.gaitpost.2006.04.014

Ricciardi, E., Bonino, D., Gentili, C., Sani, L., Pietrini, P., \& Vecchi, T. (2006). Neural correlates of spatial working memory in humans: A functional magnetic imaging study comparing visual and tactile processes. Neuroscience, 139, 339-349.

Riley, M. A., Mitra, S., Saunders, N., Kiefer, A. W., \& Wallot, S. (2012). The interplay between postural control and memory for spatial locations. Experimental Brain Research. 217, 4352. doi: 10.1007/s00221-011-2970-y

Risey, J., \& Briner, W. (1990). Dyscalculia in patients with vertigo. Journal of Vestibular Research: Equilibrium \& Orientation, 1, 31-37.

Rudkin, S. J., Pearson, D. G., \& Logie, R. H. (2007). Executive processes in visual and spatial working memory tasks. The Quarterly Journal of Experimental Brain Psychology, 60, 79-100.

Smith, P. F., Darlington, C. L., \& Zheng, Y. (2010). Move it or lose it - Is stimulation of the vestibular system necessary for normal spatial memory? Hippocampus, 20, 36-43. doi:10.1002/hipo.20588

Stein, M. \& Robinski, M. (2012). Simulator sickness in flight simulators of the German armed forces. Aviation Psychology and Applied Human Factors, 2, 11-19. doi: 10.1027/2192$0923 / \mathrm{a} 000022$

Theeuwes, J., Belopolsky, A., \& Olivers, C. N. L. (2009). Interactions between working memory, attention and eye movements. Acta Psychologica, 132, 106-14. doi:10.1016/j.actpsy.2009.01.005 
Thomas, L. E., \& Seiffert, A. E. (2010). Self-motion impairs multiple-object tracking. Cognition, 117, 80-86. 10.1016/j.cognition.2010.07.002

Thomas, L. E., \& Seiffert, A. E. (2011). How many objects are you worth? Quantification of the self-motion load on multiple object tracking. Frontiers in Psychology, 2, 1-5. doi: 10.3389/fpsyg.2011.00245

Tresch, M. C., Sinnamon, H. M., \& Seamon, J. G. (1993). Double dissociation of spatial and object visual memory: evidence from selective interference in intact human subjects. Neuropsychologia, 31, 211-9.

VanderVelde, T. J., Woollacott, M. H., \& Shumway-Cook, A. (2005). Selective utilization of spatial working memory resources during stance posture. Cognitive Neuroscience and Neuropsychology, 16, 773-777.

Vergauwe, E., Barrouillet, P., \& Camos, V. (2009) Visual and spatial working memory are not that dissociated after all: A time-based resource-sharing account. Journal of Experimental Psychology: Learning, Memory, and Cognition, 35, 1012-1028. doi: 10.1037/a0015859

Vicari, S., Bellucci, S., \& Carlesimo, G. A. (2003). Visual and spatial working memory dissociation: evidence from Williams syndrome. Developmental Medicine \& Child Neurology, 45, 269-273.

Webb, C. M., Estrada, A., Kelley, A. M., Ramiccio, J. G., Rath, E., Reeves, E. R., Hill, M. E., Crivello, M. J., \& Jones, H. D. (2011). The effect of spatial disorientation on working memory and mathematical processes. The United States Army Aeromedical Research Laboratory, Warfighting Performance and Health Division, Report No. 2011-08.

Wertheim, A. H. (1998). Working in a moving environment. Ergonomics, 41, 1845-1858. 
Woodman, G. F., Vecera, S. P., \& Luck, S. J. (2003). Perceptual organization influences visual working memory. Psychonomic Bulletin \& Review, 10, 80-87

Woollacott, M., \& VanderVelde, T. J. (2008). Non-visual spatial tasks reveal increased interactions with stance postural control. Brain Research, 1208, 95-102. 10.1016/j.brainres.2008.03.005

Yardley, L., Gardner, M., Lavie, N., \& Gresty, M. (1999). Attentional demands of perception of passive self-motion in darkness. Neuropsychologia, 37, 1293-1301.

Yardley, L., Papo, D., Bronstein, A., Gresty, M., Gardner, M., Lavie, N., \& Luxon, L. (2002). Attentional demands of continuously monitoring orientation, using vestibular information. Neuropsychologia, 40, 373-383.

Zimmer, H. D., Munzer, S., \& Umla-Runge, K. (2010). Visuo-spatial Working Memory as a Limited Resource of Cognitive Processing. In M. W. Crocker \& J. Siekmann (Eds.), Resource-Adaptive Cognitive Processes (pp. 13-34). Berlin, Heidelberg: Springer Berlin Heidelberg. doi:10.1007/978-3-540-89408-7 
Appendices 


\section{Appendix A: Participant Informed Consent}

\section{Informed Consent Form}

Study: Effects of Motion Cuing on Visuo-Spatial Working Memory Tasks

Faculty Sponsor: Dr. Chris Herdman, Department of Psychology, Carleton University, tel. 520-2600 x. 8122

The purpose of this informed consent form is to ensure that you understand both the purpose of the study and the nature of your participation. The informed consent must provide you with enough information so that you have the opportunity to determine whether you wish to participate in the study. This study has received clearance by the Carleton University Psychology Research Ethics Board (Ethics Approval: 13134). Please ask the researcher to clarify any concerns that you may have after reading this form.

Research Personnel: In addition to the Faculty Sponsor named above, the following people are involved in this research and may be contacted at any time should you require further information about this study:

\begin{tabular}{|c|c|c|c|c|}
\hline Name & Title & Department & Email & $\underline{\text { Phone }}$ \\
\hline Chris Nicholson & MA Student & Psychology & Chris_nicholson@carleton.ca & $520-2600 \times .2487$ \\
\hline
\end{tabular}

Other Contacts: Should you have any ethical concerns regarding this study, please contact Dr. Shelley Brown. For any other concerns about this study, please contact Dr. Anne Bowker.

\begin{tabular}{|c|c|c|}
\hline Name & Position & Phone \\
\hline Dr. Shelley Brown & Chair, Psychology Research Ethics Board & $520-2600$ x. 1505 \\
\hline Dr. Anne Bowker & Chair, Department of Psychology & $520-2600 \times$ x. 8218 \\
\hline
\end{tabular}

Purpose: The purpose of this study is to determine how motion affects visuo-spatial working memory tasks.

Task: In this study, you will be harnessed in a motion seat positioned in front of a large-screen monitor. Two objects will appear on the monitor. You will be asked to remember either the location or the visual characteristics of these objects for 15 seconds. On some of the trials, your seat will move during these 15 seconds. Whenever the seat moves, please indicate whether the seat moved left or right by pressing the appropriate button. After 15 seconds have elapsed, two objects will appear on the screen. Your task is to indicate whether these two objects are the same or different from the first two objects you saw. If you were asked to remember the location of the objects, then respond "same" if the two objects appear in the same location as the objects in the first display (even if the visual characteristics of the objects are different). If your task was to remember the visual characteristics of the objects, then respond "same" if the two objects have the same visual characteristics as the objects in the first display (even if the location of the objects are different). The experiment consists of 4 blocks of 30 trials each, for a total of 120 trials. If required, you can take a break between each block of trials.

Duration, Locale \& Compensation: Testing will take place in VSIM 2210 and will take approximately one hour. You will receive $1.0 \%$ credit for your participation.

Potential Risks/Discomfort: There are no potential psychological risks associated with participation in this experiment. Please note that your performance on the task in this experiment does not provide an indication of your suitability for university studies. However, if you feel anxious and/or uncomfortable about your performance, or if you begin to feel sick, then please bring your concerns to the researcher's attention immediately.

Anonymity/Confidentiality: All data collected in this experiment will be kept strictly confidential through the assignment of a coded number and securely stored on a local computer for a maximum of ten 
years. Similarly, this Informed Consent form will be kept for a maximum of ten years before being destroyed. The information provided will be used for research purposes only. You will not be identified by name in any reports produced from this study. Further, the information is made available only to the researchers associated with this experiment

Right to Withdraw/Omit: You have the right to withdraw from this experiment at any time without academic penalty. Your participation in this experiment is completely voluntary.

I have read the above description of the study assessing the impact of motion cuing on visuo-spatial working memory tasks. By signing below, this indicates that I agree to participate in the study, and this in no way constitutes a waiver of my rights.

Name:

Signature:
Date:

Witness: 
Appendix B: Participant Demographic Questionnaire

Participant Demographic Questionnaire

Effects of Motion Cuing on Visuo-Spatial Working Memory Tasks

Gender

Male $\square \quad$ Female $\square$

Age 


\section{Appendix C: Participant Debrief}

\section{Effects of Motion Cuing on Visuo-Spatial Working Memory Tasks}

Thank you for your participation in this study. This study is designed to investigate how your body's perception of motion affects your ability to remember the location and identity of visual information. Research in the working memory literature has provided evidence that there are two distinct systems responsible for the storage of visual (identity-based) and spatial (location-based) information. It is hypothesized here that the task of responding to motion cues will selectively interfere with the storage of location-based information and not with the storage of identifybased information. If this pattern of data is observed, then it will bolster the argument for the separability of visual and spatial storage. Furthermore, this finding would have practical implications for the use of motion cuing in simulators used to train drivers and pilots, insofar as driving a car and piloting an airplane both rely on spatial working memory. This experiment is the first in a series of experiments that will explore this line of research. If you are interested in learning more about visuo-spatial working memory, then please see the following:

Darling, S., Sala, D. S., \& Logie, R. H. (2009). Dissociation between appearance and location within visuo-spatial working memory. The Quarterly Journal of Experimental Psychology, 62, 417-425.

Logie, R. H. (2011). The functional organization and capacity limits of working memory. Current Directions in Psychology, 20, 240-245.

This study has received clearance by the Carleton University Psychology Research Ethics Board (Ethics Approval: 13-134). Should you have any ethical concerns regarding this study then please contact Dr. Shelley Brown (Chair, Psychology Research Ethics Board, 520-2600 ext. 1505 ). Should you have any other concerns about this study then please contact Dr. Anne Bowker, (Chair, Department of Psychology, 613-520-2600 ext. 8218) or any of the following individuals:

\begin{tabular}{|c|c|c|c|c|}
\hline Name & Title & Department & $\underline{\text { Study Role }}$ & $\underline{\text { Contact Info. }}$ \\
\hline Chris Nicholson & MA Student & Psychology & Principal Researcher & Chris_nicholson@carleton.ca \\
\hline Dr. Chris Herdman & Professor & Psychology & Faculty Advisor & 520-2600 x. 8122 \\
\hline
\end{tabular}

